\title{
Quantitative estimation of cyclotide-induced bilayer membrane disruption by lipid extraction with mesoscopic simulation
}

Karina van den Broek [a, b], Matthias Epple ${ }^{[a]}$, Lisa Sophie Kersten [b], Hubert Kuhn [c] and Achim Zielesny * [b]

[a] Inorganic Chemistry and Center for Nanointegration Duisburg-Essen (CeNIDE), University of Duisburg-Essen, Essen, Germany

[b] Institute for Bioinformatics and Chemoinformatics, Westphalian University of Applied Sciences, Recklinghausen, Germany

D-45665 Recklinghausen, August-Schmidt-Ring 10

*Correspondence: achim.zielesny@w-hs.de

[c] CAM-D Technologies GmbH, Solingen, Germany

\section{E-Mail:}

vandenbroek.ka@gmail.com

matthias.epple@uni-due.de

lisa.s.kersten@studmail.w-hs.de

kuhn@molecular-dynamics.de

achim.zielesny@w-hs.de 


\section{Abstract}

Cyclotide-induced membrane disruption is studied at the microsecond timescale by Dissipative Particle Dynamics (DPD) to quantitatively estimate a kinetic rate constant for membrane lipid extraction with a "sandwich" interaction model where two bilayer membranes enclose a cyclotide/water compartment. The obtained bioactivity trends for cyclotides Kalata B1, Cycloviolacin $\mathrm{O} 2$ and selected mutants with different membrane types are in agreement with experimental findings: For all membranes investigated, Cycloviolacin $\mathrm{O} 2$ shows a higher lipid extraction activity than Kalata B1. The presence of cholesterol leads to a decreased cyclotide activity compared to cholesterol-free membranes. Phosphoethanolamine-rich membranes exhibit an increased membrane disruption. A cyclotide's "hydrophobic patch" surface area is important for its bioactivity. A replacement of or with charged amino acid residues may lead to super-mutants with above-native activity but without simple charge-activity patterns. Cyclotide mixtures show linearly additive bioactivities without significant sub- or over-additive effects. The proposed method can be applied as a fast and easy-to-use tool for exploring structureactivity relationships of cyclotides/membrane systems: With the open software provided, the rate constant of a single cyclotide/membrane system can be determined in about one day by a scientific end-user without programming skills. 


\section{Graphical abstract}

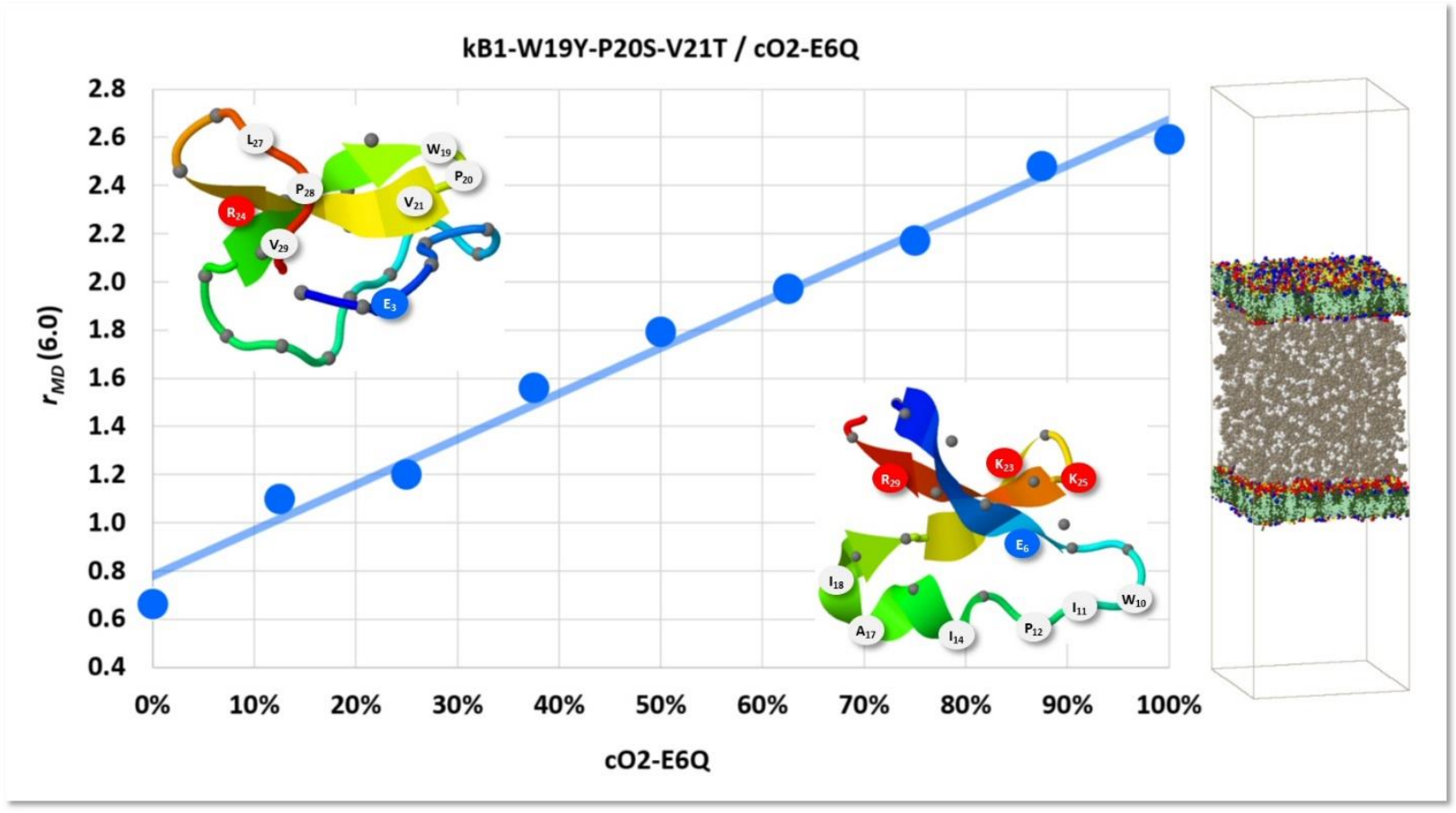

\section{Keywords}

Molecular simulation, mesoscopic simulation, Dissipative Particle Dynamics, DPD, Molecular Fragment Dynamics, MFD, phospholipid, cholesterol, cyclotide, Kalata B1, Cycloviolacin O2, mutant, DMPC, DOPE, DOPS, PIP2, SM, bilayer membrane, plasma membrane, structure-activity relationship, SAR, lipid extraction. 


\section{Introduction}

Cyclotides are plant-derived macrocyclic peptides of about 30 amino acids with their $\mathrm{N}$ - and C-termini being connected by a peptide bond. They contain a characteristic structural motif, the cyclic cystine knot (CCK), and can be divided into the Möbius and Bracelet subfamilies by a particular twist of their cyclic backbone (as well as a third main subgroup of trypsin inhibitor cyclotides which are not taken into account in this work). Due to their cyclic structure with three additional disulfide bridges cyclotides possess extraordinarily stable spatial conformations [1]. Over the last two decades cyclotides attracted increasing attention due to their interesting range of biological activities (like cytotoxic, hemolytic, anti-HIV, anti-cancer, anthelmintic, anti-fouling, antimicrobial or insecticidal effects) with a potential use as drug scaffolds and therapeutic agents. An overview of relevant studies was recently provided by a comprehensive review [2].

Cyclotide bioactivities may at least partially be traced to specific interactions with lipid bilayer membranes [3-34] which have also been demonstrated by simulation models [3538]: Whereas a single or a few cyclotide peptides already interact with membranes they do neither impair their structural integrity nor their segregating function. However, a collective interaction of multiple cyclotides is able to distort up to disrupt the bilayer structure. Different modes of action depending on the cyclotide/membrane system are to be expected: These comprise cyclotide membrane binding $[5,6,8-10,12,14,15,17-19$, $21,23-25,36]$ and intrusion [27, 31, 32, 34] as well as cell penetration [6, 21, 27, 32, 30] up to the final ability to disrupt a membrane by a postulated formation of pores [3, 5-7, 9 , $11,13,14,16,17,19-24,26,29,31,35-38]$ or by distinct lipid extraction [17, 35, 37, 38].

Lipid extraction as a particular mechanism for membrane disruption was experimentally shown in [17] as well as detected by coarse-grained Molecular Dynamics (MD) [35, 38] and mesoscopic [37] simulations. In [35] a detailed interfacial bioactivity model is described for cyclotide Kalata B1 where two configurations of Kalata B1 oligomers, denoted tower-like and wall-like clusters, are formed. Conjugation between wall-like clusters results in the formation of a ring-like hollow on the membrane surface. This interfacial membrane binding of Kalata B1 induces a positive membrane curvature 
with a subsequent lipid extraction from the membrane through the ring-like hollow into the Kalata B1 cluster.

This work is based on previous qualitative findings in [37] concerning cyclotide-induced lipid extraction and attempts to quantify this aspect of cyclotide bioactivity by mesoscopic simulation with Dissipative Particle Dynamics (DPD). The proposed geometrical simulation setup for quantitative analysis resembles a "sandwich" where two bilayer membranes enclose a cyclotide/water compartment: Within this compartment a kinetic rate of cyclotide-induced membrane disruption by lipid extraction (abbreviated $r_{M D}$ ) is evaluated at the microsecond timescale. Quantitative $r_{M D}$ estimates are determined for a range of different cyclotide/membrane systems containing the Möbius cyclotide Kalata B1 (abbreviated kB1), Bracelet cyclotide Cycloviolacin O2 (abbreviated cO2) as well as some of their particular mutants and compared to experimental findings.

\section{Methods and simulation setup}

\subsection{Dissipative Particle Dynamics}

Dissipative Particle Dynamics (DPD) is a mesoscopic simulation technique for isothermal complex fluids and soft matter systems. It satisfies Galilean invariance and isotropy, conserves mass and momentum and achieves a rigorous sampling of the canonical NVT ensemble due to soft particle pair potentials that diminish molecular entanglements or caging effects. DPD is expected to show correct hydrodynamic behavior and to obey the Navier-Stokes equations [39-43]. DPD particle trajectories are guided by Newton's equation of motion where the total force on a particle exerted by other particles consists of a conservative, a dissipative (frictional) and a random part. The opposing dissipative and random forces depend on each other and act as a thermostat conserving the total momentum and introducing Brownian motion into the system. The conservative forces comprise soft DPD particle repulsions as well as possible harmonic springs between bonded and electrostatic interactions between charged particles. Dissipative DPD forces depend 
on relative particle velocities so that the common MD Velocity-Verlet integration of the equations of motion has to be modified [43].

DPD particles in general may be arbitrarily defined as "fluid packets" [40]. "Molecular Fragment" Dissipative Particle Dynamics is a "bottom-up" DPD variant [37, 43-49] that identifies each particle with a distinct small molecule of molar mass in the order of $100 \mathrm{Da}$. Larger molecules are composed of adequate smaller "molecular fragment" particles that are bonded by harmonic springs to mimic covalent connectivities and spatial 3D conformations (compare figure 1).

\subsection{Open simulation software}

All simulations of this work were performed with the open mesoscopic simulation environment MFsim [50] using the default open DPD engine Jdpd [51] where all illustrations and animations were derived from MFsim graphics. MFsim implements the SPICES particle structure line notation [52] and provides a peptide/protein editor with automated SPICES decomposition from PDB files [53]. A compartment editor supports the initial setup of interaction models by defined molecule placement in the simulation box including spatial peptide/protein 3D structures.

\subsection{Particle data}

All particle related data used for the simulations of this work are provided in particle set text file ParticleSet_AA_V03.txt [54]. They are derived from [37] with rescaled molecular volumes (via MFsim menu function Rescale to new V(min) with water being the smallest particle of volume $30 \AA^{3}$ ) and rescaled particle-particle repulsions (via MFsim menu function Rescale repulsions a(ij) with default settings). Particle set text file section Particle description contains all particle related definitions, section Particle interactions lists the mutual particle-particle repulsions that guide the DPD dynamics and section Amino acids provides peptide/protein decomposition related information. 


\subsection{Simulation details}

The definition and execution of MFsim simulation jobs used for this work is described by a tutorial which may be downloaded from [55] or viewed/downloaded in animated form at [56]. In addition, the simulation job definitions for the studied cyclotide/membrane systems are openly available as MFsim archive ZIP files at [57]: They may be imported into MFsim and reviewed in detail (e.g. with running MFsim on a standard notebook computer) as well as ubiquitously reproduced with MFsim running on a multicore workstation using 8 parallelized Jdpd calculation threads (defined in MFsim via Preferences/Parallel computing/Parallel calculators).

For all simulations MFsim default settings are used if not denoted otherwise. All molecular species are manually defined by their SPICES strings. The cyclotide SPICES strings are automatically derived from their corresponding PDB files and additional settings: Mutations and amino acid sequence loop closures are manually defined, disulfide bridges and $\mathrm{pH}$ dependent charges are assigned in an automated manner. All amino acid side chains are charged for $\mathrm{pH}$ 7.4. The "sandwich" model start geometry (see below) is constructed with xy layer compartments: Single water particles are randomly positioned within their compartments (but outside of exclusive cyclotide spheres, see below). Phospholipids and cholesterol are collapsed and compressed into linear SPICES tubes according to their start/end tagged particles [52] and randomly positioned in the xy plane perpendicular to their tube orientation with their start particles being located at the compartment's upper or lower z-surface. All amino acid sidechain particles of the cyclotides are collapsed onto their corresponding backbone particles which themselves are relatively positioned according to the $\mathrm{C}_{\alpha}$ coordinates defined in their PDB 3D structures. These collapsed cyclotide 3D particle structures are then squeezed and randomly rotated within exclusive spheres (with a DPD volume according to the cyclotide particle count) and randomly positioned in their compartment without overlap. Different random start geometries are generated with different Geometry random seed values. Protein distance forces for preservation of the cyclotide backbone 3D structure during simulation are completely activated with force constants (denoted $k_{B B}$ ) of 4 DPD units for all cyclotides. Molecule backbone forces are defined for cholesterol with a force 
constant of 4 DPD units (see below and figure 1). For integration of the equations of motion Shardlow's S1 scheme [58, 59] is used (denoted S1MVV in MFsim setting Integration type). Periodic boundaries are turned on in $\mathrm{x}$ and $\mathrm{y}$ direction with reflective box walls in $z$ direction. Each simulation is preceded by a conservative energy minimization procedure with 100 Initial minimization steps to relax the initial "high energy" box geometry (particularly due to the collapsed and squeezed SPICES tubes and cyclotide 3D structures). For additional initial system relaxation - now in contact with a "293 K thermostat" - each simulation starts with 100 Initial velocity scaling steps that also remove "excess momentum". The molecular ensembles for the simulation runs comprise more than 600,000 particles representing more than two million atoms and are studied with up to 125,000 simulation steps which correspond to a physical simulation time of $7.5 \mu \mathrm{s}$. As a rule of thumb, a single simulation run with 8 parallelized Jdpd calculation threads allows to study the dynamics for several microseconds in less than a day with an ordinary multicore workstation.

\subsection{Molecular fragmentation}

Figures 1 and 2 sketch the molecular structure decompositions of the bilayer membrane constituent phospholipids and cholesterol into connected "molecular fragment" particles using the SPICES line notation. In general, adequate "molecular fragment" particles have to be determined by experience and chemical intuition due to the lack of an objective fragmentation procedure (e.g. see their "circular identification" in figure 1 on the left). In order to keep spatial characteristics of a molecular species it may be necessary to impose additional intramolecular "backbone forces" between specific particles, e.g. the "rod-like" geometry of cholesterol due to its rigid ring system is reflected by an additional "molecule backbone force" spring as sketched in figure 1 on the right (where the SPICES line notation supports the corresponding backbone labels). 

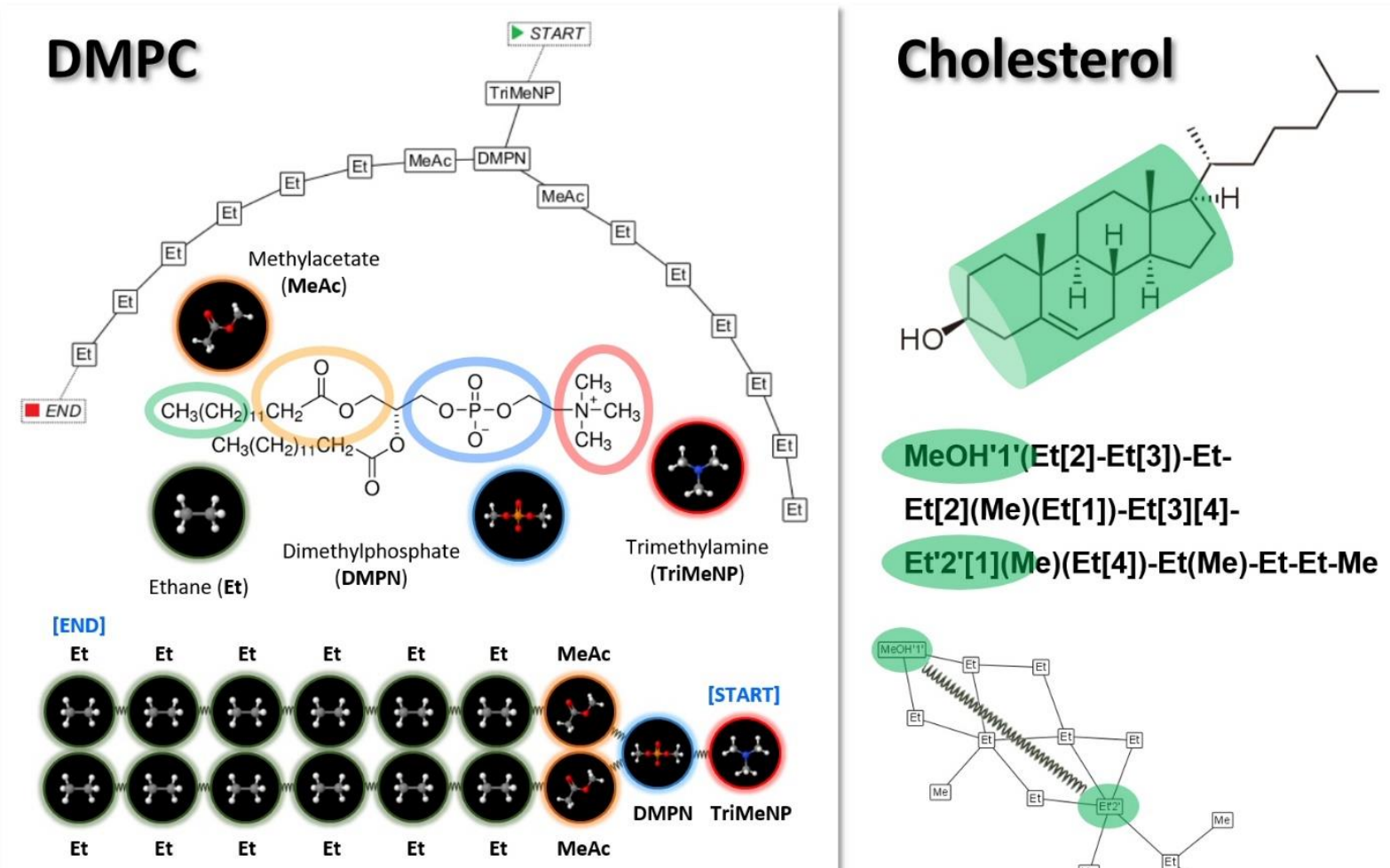

TriMeNP[START]-DMPN(MeAc-6Et)(MeAc-6Et[END])

MeOH'1'(Et[2]-Et[3])-Et-

$\mathrm{Et}[2](\mathrm{Me})(\mathrm{Et}[1])-\mathrm{Et}[3][4]-$

Et'2'[1](Me)(Et[4])-Et(Me)-Et-Et-Me

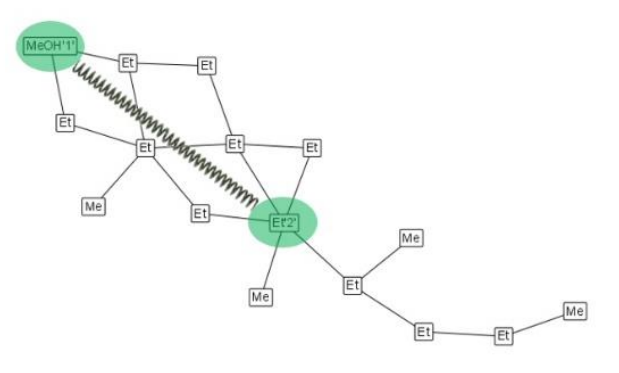

Figure 1. Left: Decomposition of phospholipid DMPC into "molecular fragment" particles (ethane, abbreviated with "particle acronym" Et, methyl acetate, MeAc, negatively charged dimethyl phosphate, DMPN, and positively charged trimethylamine, TriMeNP) and their adequate topological connection with harmonic springs. At the top the topological particle connection illustration generated by MFsim is depicted with the corresponding SPICES line notation shown at the bottom (the included start and end tags support adequate spatial positioning in the simulation box for an initial bilayer geometry [52]). Right: "Molecular fragment" particle decomposition of cholesterol allowing for an adequate spring force definition between the highlighted particles $\mathrm{MeOH}$ and $\mathrm{Et}$ (with backbone labels ' 1 ' and ' 2 ') to account for the spatial rigidity of the cholesterol ring system. Particle acronyms follow the conventions defined in the particle set text file. 


\section{Cholesterol}

(3ß)-Cholest-5-en-3-ol

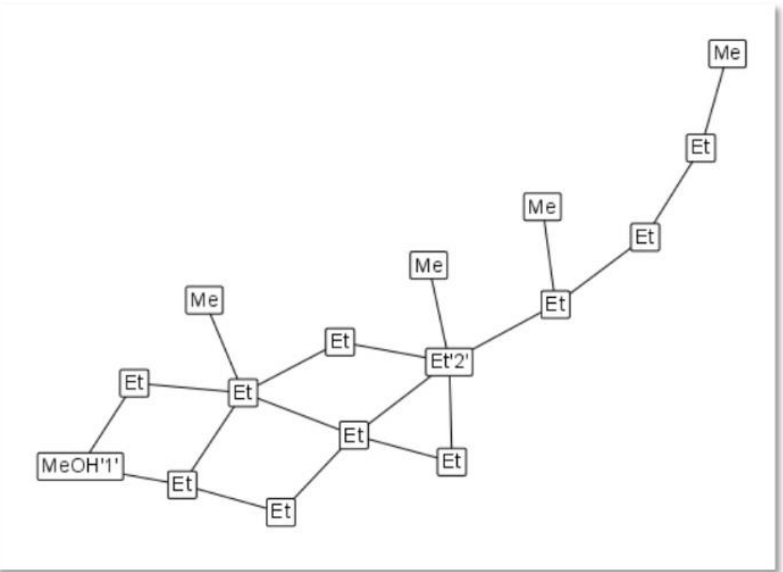

MeOH'1'(Et[2]-Et[3])-Et-Et[2](Me)(Et[1])Et[3][4]-Et'2'[1](Me)(Et[4])-Et(Me)-Et-Et-Me

\section{DOPE}

1,2-Dioleoyl-sn-glycero-3phosphoethanolamine

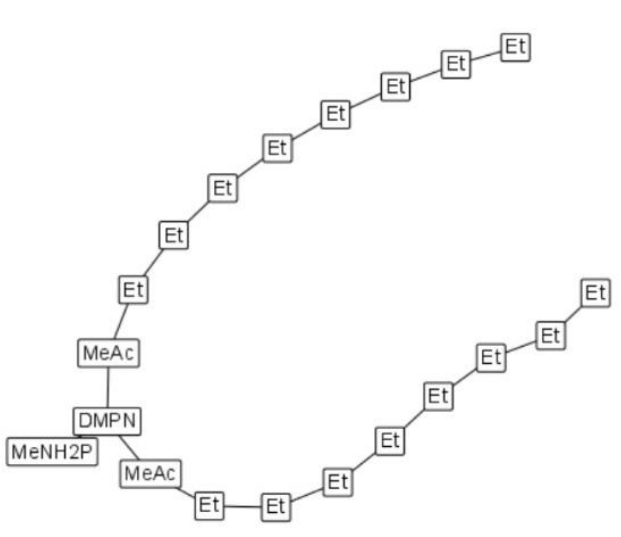

MeNH2P-DMPN(MeAc-8Et)(MeAc-8Et)

\section{DMPC}

1,2-Dimyristoyl-sn-glycero-3-phosphocholine

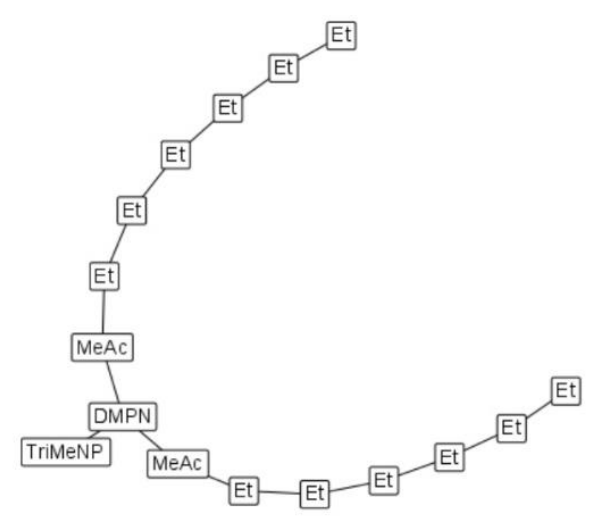

TriMeNP-DMPN(MeAc-6Et)(MeAc-6Et)

\section{PIP $_{2}$}

Phosphatidylinositol 4,5-bisphosphate

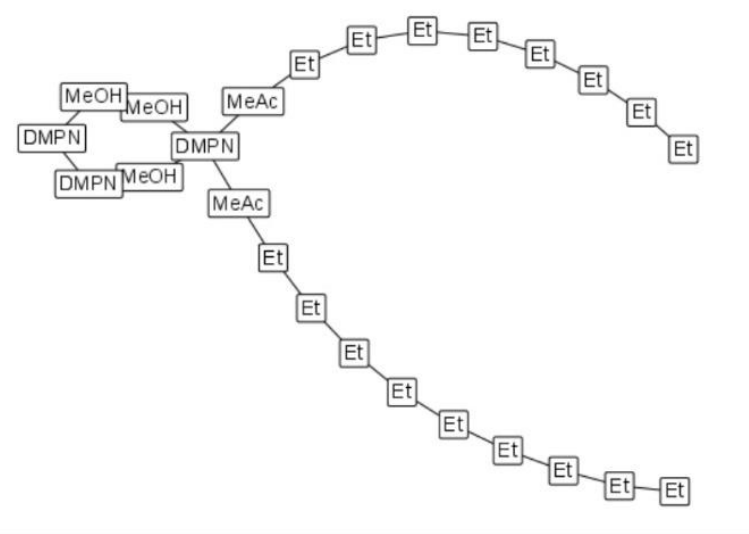

DMPN[1](MeAc-8Et)(MeAc-9Et)-MeOHDMPN-DMPN-MeOH-MeOH[1] 
DOPS

1,2-Dioleoyl-sn-glycero-3-phospho-L-serine

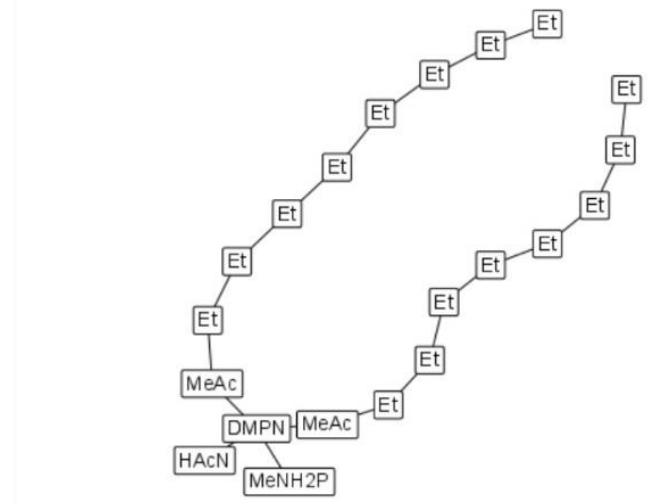

HAcN-DMPN(MeNH2P)(MeAc-8Et)(MeAc-8Et)
SM

$\mathrm{N}$-(Hexadecanoyl)-sphing-4-enine-1phosphocholine

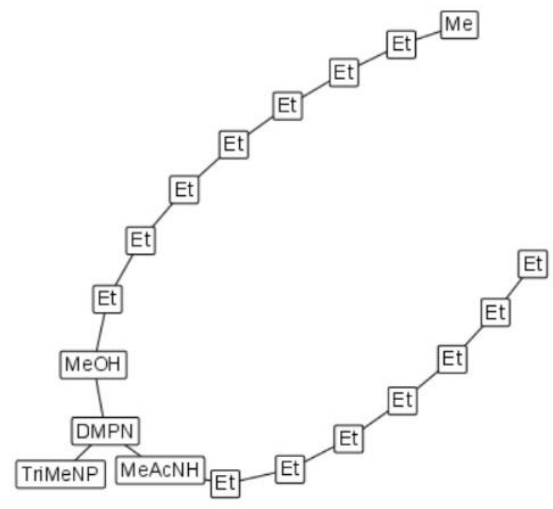

TriMeNP-DMPN(MeAcNH-7Et)(MeOH-7Et-

$\mathrm{Me})$

Figure 2. "Molecular fragment" particle decomposition of the constituent membrane lipids (acronym, chemical name, topological particle connection illustration generated by MFsim and corresponding SPICES line notation). Particle acronyms follow the conventions defined in the particle set text file.

Figure 3 summarizes the decomposition of cyclotides kB1 (PDB ID: 1NB1) and cO2 (PDB ID: 2KNM) into "molecular fragment" particles. The cyclotides consist of a loop structure with 29 (kB1)/30 (cO2) amino acids where the "hydrophobic patch" as a specific structural feature is highlighted. The "molecular fragment" particle decomposition is generated by the automated PDB-file-based fragmentation procedure of MFsim. For this purpose, section Amino acids of the particle set text file defines SPICES strings for all 20 proteinogenic amino acids and $\mathrm{pH}$ dependent (charged) particle replacements for the $\mathrm{C}$ - and $\mathrm{N}$-terminus as well as the sidechains (note that in figure 3 only the sidechain replacements for $\mathrm{pH} 7.4$ signaled by the amino acid tags $\{S-\} /\{S+\}$ are considered since both cyclotides contain a loop structure). Additional intramolecular "protein distance forces" between backbone particles are defined for control of the rigidity/flexibility of the spatial 3D backbone structure. The number label of a single cyclotide amino acid is derived from Jmol [60] labelling which is used by MFsim. 


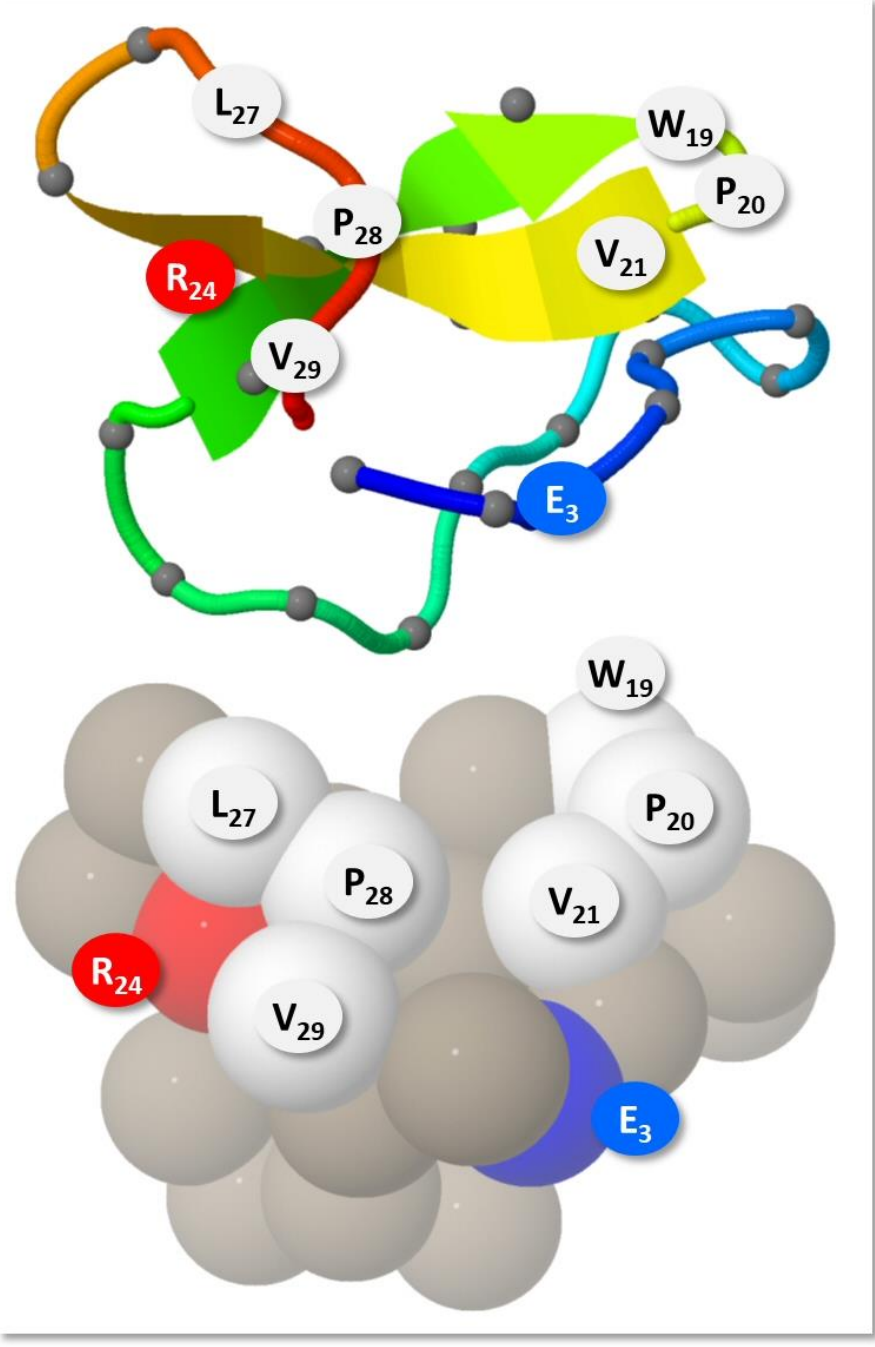

\begin{tabular}{|c|c|}
\hline$C\left[{ }^{*}\right][1]$ & MeAcNHBB[4](MeSHSS[1]) \\
\hline G & $-\mathrm{MeAcNHBB}$ \\
\hline$E_{3}\{S-\}$ & -MeAcNHPD2(Me-HAcN) \\
\hline $\mathbf{T}$ & $-\mathrm{MeAcNHBB}(\mathrm{PrOH})$ \\
\hline$C[2]$ & -MeAcNHBB(MeSHSS[2]) \\
\hline V & $-\mathrm{MeAcNHBB}(\mathrm{Pr})$ \\
\hline G & $-\mathrm{MeAcNHBB}$ \\
\hline G & $-\mathrm{MeAcNHBB}$ \\
\hline $\mathbf{T}$ & -MeAcNHBB(PrOH) \\
\hline$C[3]$ & -MeAcNHBB(MeSHSS[3]) \\
\hline $\mathbf{N}$ & $-\mathrm{MeAcNHBB}(\mathrm{AcNH} 2)$ \\
\hline $\mathbf{T}$ & -MeAcNHBB(PrOH) \\
\hline $\mathbf{P}$ & -AzolidBB \\
\hline G & -MeAcNHBB \\
\hline$C[1]$ & -MeAcNHBB(MeSHSS[1]) \\
\hline $\mathbf{T}$ & -MeAcNHBB(PrOH) \\
\hline $\mathrm{C}[2]$ & -MeAcNHBB(MeSHSS[2]) \\
\hline $\mathbf{S}$ & $-\mathrm{MeAcNHBB}(\mathrm{MeOH})$ \\
\hline $\mathbf{W}_{19}$ & -MeAcNHPD1(Me-Pyrrole-Ph) \\
\hline $\mathbf{P}_{20}$ & -AzolidPD1 \\
\hline $\mathbf{V}_{21}$ & -MeAcNHPD1(Pr) \\
\hline$C[3]$ & -MeAcNHBB(MeSHSS[3]) \\
\hline $\mathbf{T}$ & -MeAcNHBB(PrOH) \\
\hline $\mathbf{R}_{24}\{\mathbf{S}+\}$ & -MeAcNHPD3(Pr-GuanidineP) \\
\hline $\mathbf{N}$ & $-\mathrm{MeAcNHBB}(\mathrm{AcNH} 2)$ \\
\hline G & $-\mathrm{MeAcNHBB}$ \\
\hline $\mathbf{L}_{27}$ & -MeAcNHPD1(Me-Pr) \\
\hline $\mathbf{P}_{28}$ & -AzolidPD1 \\
\hline $\mathbf{V}_{29}[*]$ & -MeAcNHPD1[4](Pr) \\
\hline
\end{tabular}




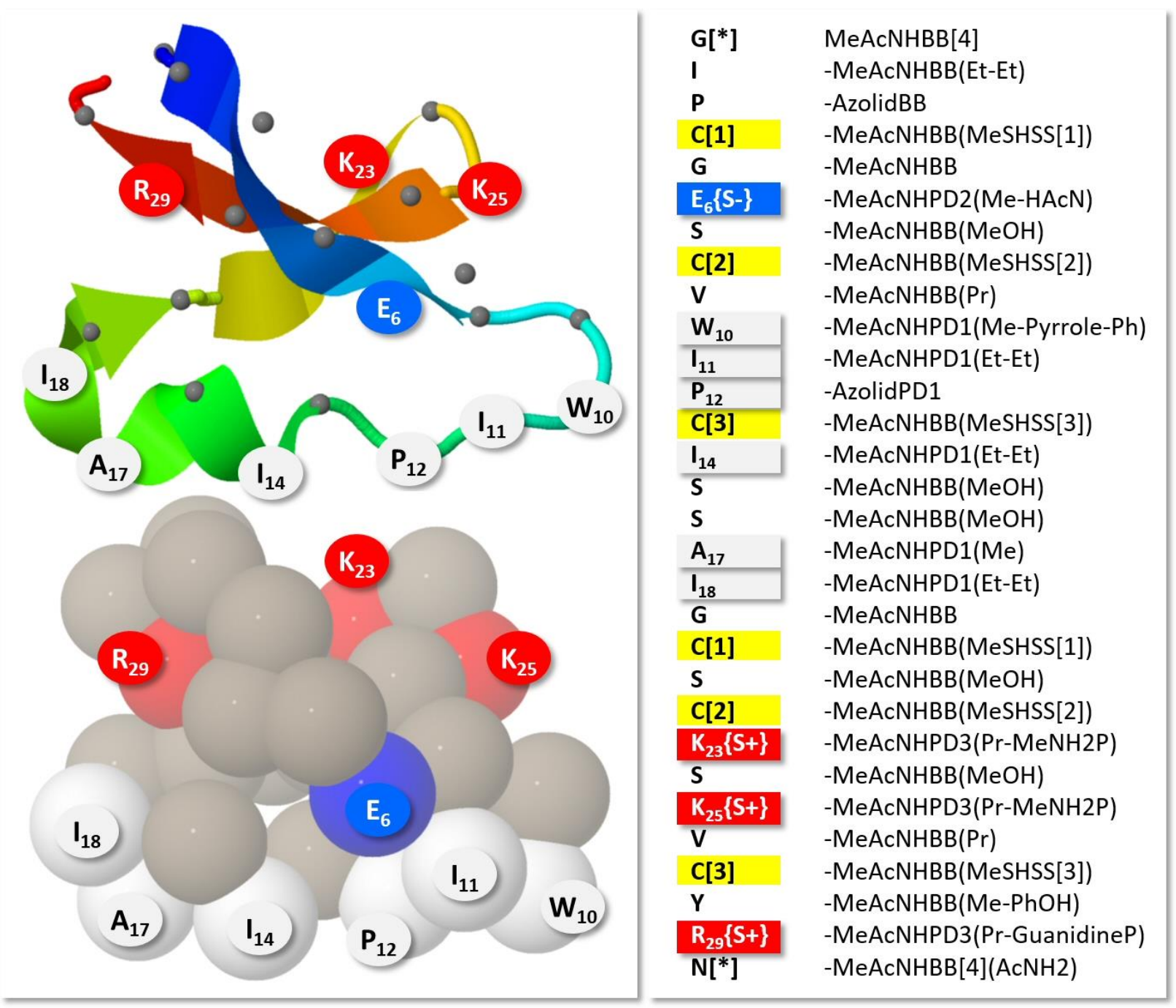

Figure 3. Cyclotides kB1 (top) and $\mathrm{cO} 2$ (bottom). Top, upper left: Cartoon representation of the $\mathrm{C}_{\alpha}$ backbone of kB1 with the 6 highlighted amino acids of the "hydrophobic patch" $\left(\mathrm{W}_{19}, \mathrm{P}_{20}, \mathrm{~V}_{21}, \mathrm{~L}_{27}, \mathrm{P}_{28}, \mathrm{~V}_{29}\right)$ and the two charged sidechain amino acids $\left(E_{3}, R_{24}\right)$. Top, lower left: Corresponding backbone particle 3D structure generated by MFsim from the 3D coordinate information of the PDB file (side chain particles are not shown) with the backbone particles of the hydrophobic patch highlighted in white and the positively/negatively charged amino acid backbone particles colored in red/blue. Top, right: Amino acid sequence (bold font type from top to bottom) with corresponding parts of the SPICES line notation generated by the automated MFsim "molecular fragment" particle decomposition for peptides and proteins. The amino acid tags $\left\{S_{+}\right\} /\{S-\}$ denote the positively/negatively charged side chain (particle) at $\mathrm{pH} 7.4$ for $\mathrm{R}_{24} / \mathrm{E}_{3}$, bracketed indices indicate connections and the bracketed asterisk signals a closed loop. "Hydrophobic patch" and charged amino acids are highlighted and labelled, cysteine in disulfide bridges is indicated by a yellow background color. All particle acronyms follow the conventions in the particle set text file (suffix BB denotes backbone particles, suffix SS particles in disulfide bridges, suffix $\mathrm{PD}<$ index> defines a "probe definition" that may be used for particle 
highlighting with specific colors. e.g. to visualize the "hydrophobic patch"). Bottom: Corresponding information for cyclotide $\mathrm{cO} 2$.

All cyclotide mutants are constructed with the MFsim peptide/protein editor which supports amino acid replacements. These replacement operations do not influence the spatial $3 \mathrm{D}$ backbone structure which is in general likely to cause structural inaccurateness in the backbone conformation. But cyclotides kB1 and $\mathrm{cO} 2$ exhibit an extraordinary stable spatial conformation (with each containing a loop structure plus 3 disulfide bridges) so that only negligible conformational backbone changes are expected - note, that all sidechain particles are flexible being only constrained by standard "spring force" bonds.

Cyclotide mutants follow the notation <original amino acid><number label of amino acid ><amino acid of mutant $>$ where multiple mutations are concatenated, e.g. mutant kB1-W19Y has a single replacement with amino acid tryptophan (W) at position 19 being replaced by amino acid tyrosine (Y), mutant kB1-W19Y-P20S-V21T contains three amino acid replacements at consecutive positions: Tryptophan (W) at position 19 is replaced by tyrosine $(\mathrm{Y})$, proline $(\mathrm{P})$ at position 20 by serine $(\mathrm{S})$ and valine $(\mathrm{V})$ at position 21 by threonine $(\mathrm{T})$.

\subsection{Bilayer membrane models}

Each bilayer membrane is constructed with 1600 phospholipid/cholesterol molecules. The phospholipid composition of plasma membrane models is chosen to be $40 \%$ DMPC, 20\% DOPE, 5\% PIP 2, 10\% DOPS and 25\% SM (see figure 2) with an inner to outer leaflet distribution of 24 to 76 for DMPC, 77.5 to 22.5 for DOPE, 75 to 25 for $\mathrm{PIP}_{2}, 1$ to 0 for DOPS (only inside) and 22 to 78 for SM [61, 62]. The proportion of cholesterol is varied from $0 \%$ to $50 \%$. If cholesterol molecules are present, the sum of phospholipid and cholesterol molecules is equalized for the inner and outer membrane leaflet. The start geometry consists of a bilayer of start/end-tag oriented phospholipid/cholesterol molecules (compare figures 1 and 4) being manually built with the compartment editor of MFsim. The $x y$ area of the simulation box can be chosen to allow for a specific area per lipid molecule [63-68]. Pure DMPC 
or DOPE bilayer membranes without cholesterol are abbreviated NoC-DMPC-M or NoC-DOPE-M respectively. A plasma membrane without cholesterol is abbreviated NoC-PM, a plasma membrane with $50 \%$ cholesterol molecules $50 \mathrm{C}-\mathrm{PM}$.

\subsection{Cyclotide/membrane interaction model}

The proposed "sandwich" cyclotide/membrane interaction model is an artificial construct to estimate lipid extraction, designed for simplicity without any additional forces or spatial constraints as well as a clear connection to experimental findings. The choice of the cyclotide number in the cyclotide/water compartment is driven by maximizing the disruptive effect with a minimum number of cyclotides - where the extremes would be a single cyclotide within the compartment (with possible membrane interaction but no disruptive lipid extraction effect) and a biologically unrealistic compartment which solely consists of cyclotides without any water. The initial simulation box setup is sketched in figure 4: There are two water compartments at top and bottom of the simulation box with 200.000 water particles each in contact with the inner membrane leaflets. The two membrane compartments are composed as described above. The outer membrane leaflets of both membranes are in contact with two buffer compartments containing 5.000 water particles each to prevent initial contact of membranes and cyclotides. The enclosed cyclotide/water compartment consists of 190,000 cyclotide and water particles distributed according to the defined cyclotide number. Within their compartment the cyclotides are randomly oriented and positioned with their spatial 3D backbone structure (option $3 D$ random in compartment editor of MFsim), all sidechain particles are initially collapsed onto their corresponding backbone particles by default.

The number of cyclotides is chosen to be 1,000 to obtain evaluable effects within simulation times of a few microseconds. This corresponds to an initial cyclotide to water ratio within the cyclotide/water compartment of 1 to 145 for Kalata B1 (a single Kalata B1 cyclotide is represented by 55 particles, so 1,000 cyclotides lead to 55,000 particles; the compartment size without buffer is fixed to 190,000 particles, so that $190,000-55,000=135,000$ water particles are added; $135,000+10,000$ buffer water particles $=145,000$ water particles for 1,000 cyclotides result in 145 water particles for every single cyclotide in the whole cyclotide/water compartment) and 1 
to 134 for Cycloviolacin O2 (where a single Cycloviolacin O2 cyclotide is represented by 66 particles) with similar values for their mutants (due to slightly different numbers of the specific cyclotide particles).

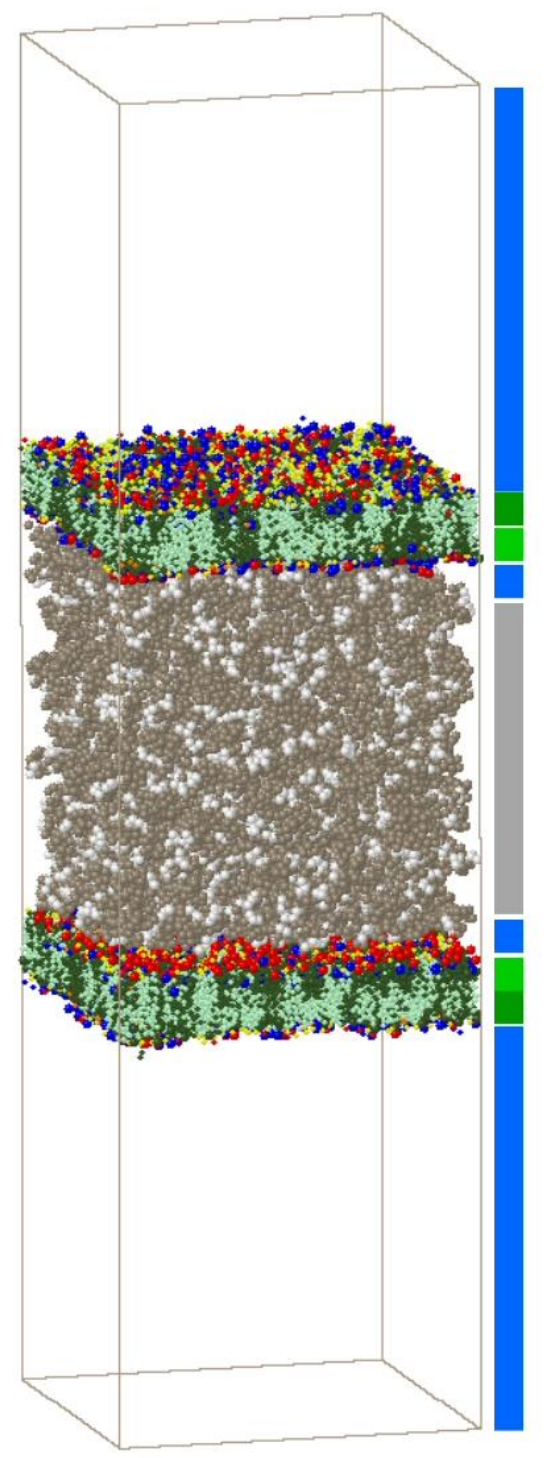

Water compartment

Inner (upper) / outer (lower) membrane leaflet Water buffer compartment

Cyclotide/water compartment

Water buffer compartment

Outer (upper) / inner (lower) membrane leaflet

Water compartment

Figure 4. Initial simulation box setup of the "sandwich" cyclotide/membrane interaction model: The backbone particles of the Kalata B1 "hydrophobic patch" are colored in white, all other backbone particles are shown in beige. The positively/negatively charged particles of the phospholipids are colored in red/blue, $\mathrm{MeOH}$ in orange and "hydrophobic" particles in olive. $\mathrm{MeOH}$ particles of cholesterol are displayed in yellow, the "hydrophobic" particles in mint. Kalata B1 sidechain particles are not shown. All water particles are omitted. 
Since the physical length of a DPD simulation box depends on the particle types and their relative numbers, the DPD lengths of the model systems containing cyclotides are adjusted to the DPD lengths of the pure membrane DPD models.

\section{Results and discussion}

\subsection{Plasma membrane models}

Plasma membrane models with areas per lipid molecule of 40,50 or $60 \AA^{2}$ build stable bilayers on the microsecond timescale. In contrast to the phospholipids which mainly stick to their initial leaflet with only rare inter-leaflet flips, cholesterol shows an extremely high inter-leaflet mobility with a steady state distribution reached after a few hundred nanoseconds. The cholesterol mobility is demonstrated at [69] for a $50 \mathrm{C}-\mathrm{PM}$ with an area per lipid of $40 \AA^{2}$. An extremely high rate of cholesterol translocation between the leaflets is also experimentally indicated [70, 71]. The overall thicknesses of the plasma membranes correspond to their different areas per lipid to be about 40 to $50 \AA$ with a hydrophobic thickness of approximately 20 to 30 $\AA$, see figure 5: The hydrophobic thickness is estimated with the width of the distribution of the hydrophobic Me and Et particles (green), the overall thickness with the distance between the inner and outer distributions of the terminal phospholipid particles (red). The charged terminal phospholipid particles form the outer water contact layer, followed by the cholesterol $\mathrm{MeOH}$ particle layer. Me and Et particles constitute the hydrophobic membrane core with a low permeability for water particles. These findings agree with experimental as well as alternative simulation results [66, 72-81]. For the studies of this work membranes with an area of $40 \AA^{2}$ per lipid are chosen. 


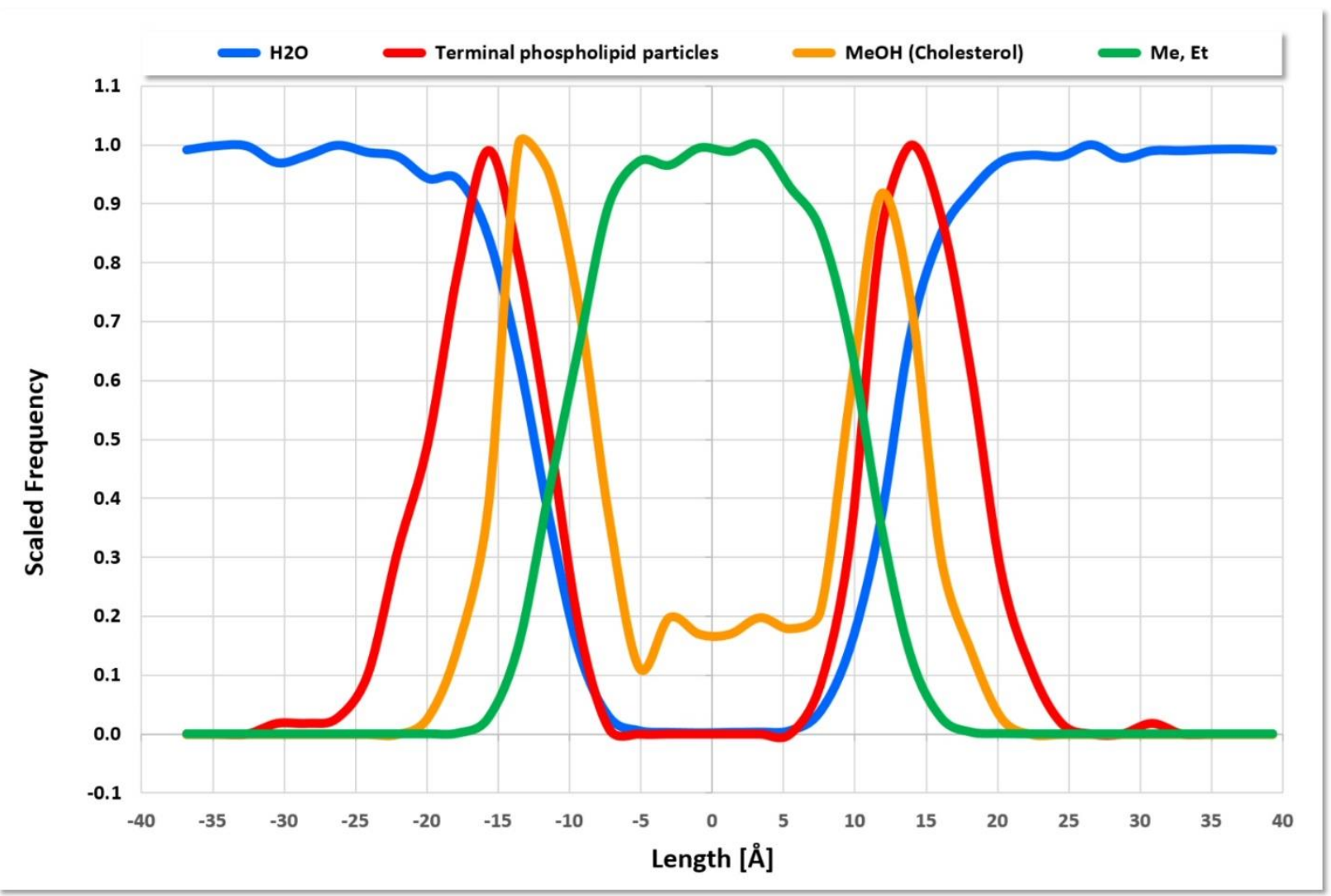

Figure 5. Snapshots of particle distributions across a 50C-PM with an area per lipid of $40 \AA^{2}$ (simulation time: $2.5 \mu \mathrm{s}$ ). The depicted length ( $\mathrm{z}$ ) axis is perpendicular to the bilayer membrane $(x y)$ plane with its origin being adjusted in the middle of the membrane, so that negative lengths correspond to the inner membrane leaflet and positive lengths to the outer one.

\subsection{Cyclotide backbone flexibility}

The backbone particle flexibility of a single Kalata B1 and a single Cycloviolacin $\mathrm{O} 2$ cyclotide within a water box is demonstrated at [82] for the specified "Protein distance forces" setting with force constants $\left(k_{B B}\right)$ of 4 DPD units. This choice allows for fast transient oscillations from the "squeezed" start geometry (due to DPD density constraints of the MFsim compartment editor) towards the 3D geometry derived from the PDB structure during the minimization process. The simulation shows an acceptable degree of backbone flexibility with an overall conservation of the imposed 3D structure. 


\subsection{Cyclotide-membrane interaction}

\section{Qualitative lipid extraction dynamics}

The qualitative cyclotide/membrane interaction behavior from the mesoscopic point of view may be summarized as follows - compare animation of the interaction of cyclotide kB1 with NoC-PM and 50C-PM at [83], figure 6 and upper row of figure 9: From the minimized start geometry the two membranes exhibit a fast crossover to the particle distributions described above. While near-membrane cyclotides may immerse into the membranes, the majority of randomly distributed cyclotides form oligomers (also described by experimental [5, 8, 9, 11, 21, 84, 85] and alternative simulation [35] studies) that themselves aggregate towards a "network structure" in the cyclotide/water compartment with emerging hydrophobic vicinities particularly created by "hydrophobic patch" amino acids. These hydrophobic vicinities are able to accommodate the hydrophobic parts of membrane lipids. Thus, a membrane lipid distribution outside the initial membranes is initiated by extraction of phospholipid/cholesterol compounds from the membranes (see highlighted circular area in figure 6). With ongoing interaction, membrane lipids successively advance into the cyclotide/water compartment which increasingly disrupts the membranes. This process is accompanied by a constant decrease of total potential energy of the system, i.e. the membrane lipid/cyclotide interaction is overall energetically preferred.

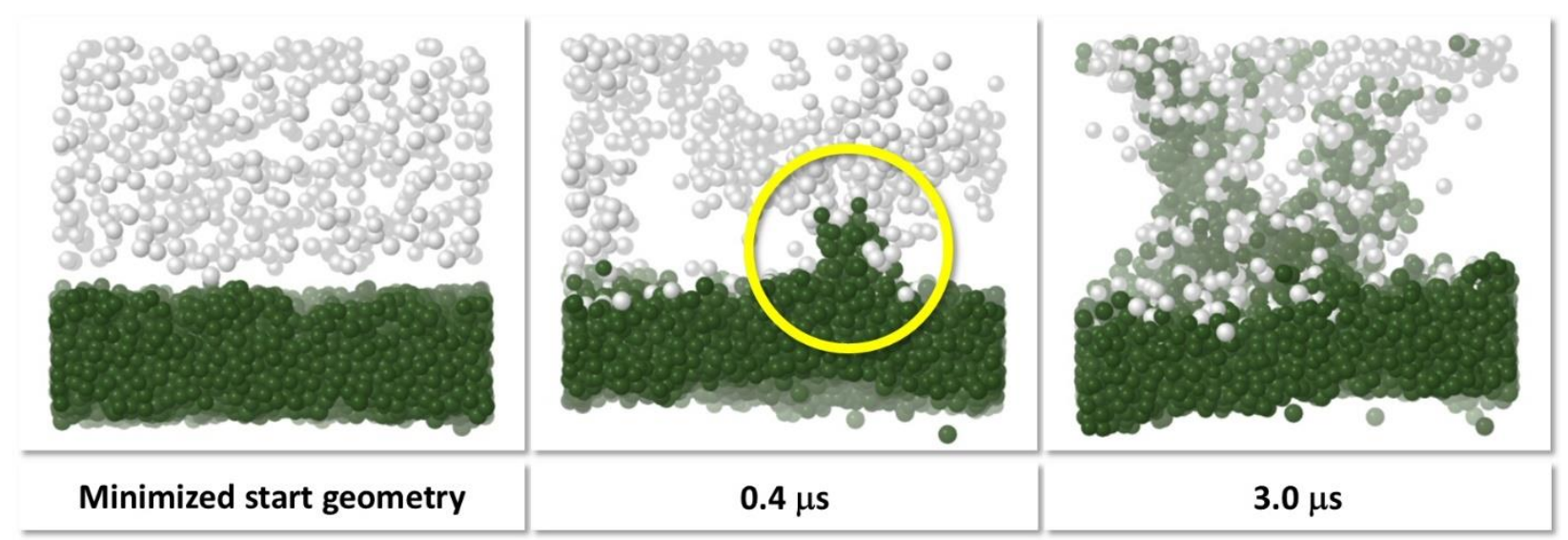

Figure 6. Interaction snapshots of kB1 with a NoC-PM at different simulation times for the same magnified simulation box cutout. Only backbone particles of the kB1 "hydrophobic patch" (white) and "hydrophobic" phospholipid particles (olive) are shown. 


\section{Quantitative lipid extraction analysis}

The quantitative analysis of the sketched membrane disruption process is performed by evaluating the temporal Et particle distribution of cholesterol and phospholipids in the cyclotide/water compartment which is initially equal to zero, i.e. there are no membrane lipids in this compartment. For that purpose the cyclotide/water compartment is partitioned into 10 equal bins along the $z$-axis with bins 2 to 9 being evaluated to avoid influence of possible membrane curvature which may affect bins 1 and 10. An animation of the temporal Et-particle-distribution evaluation is available at [86], figure 7 sketches results where the percentages on the $y$-axis refer to the corresponding total numbers of Et particles in the membranes which allows for comparability between different cyclotide/membrane systems.

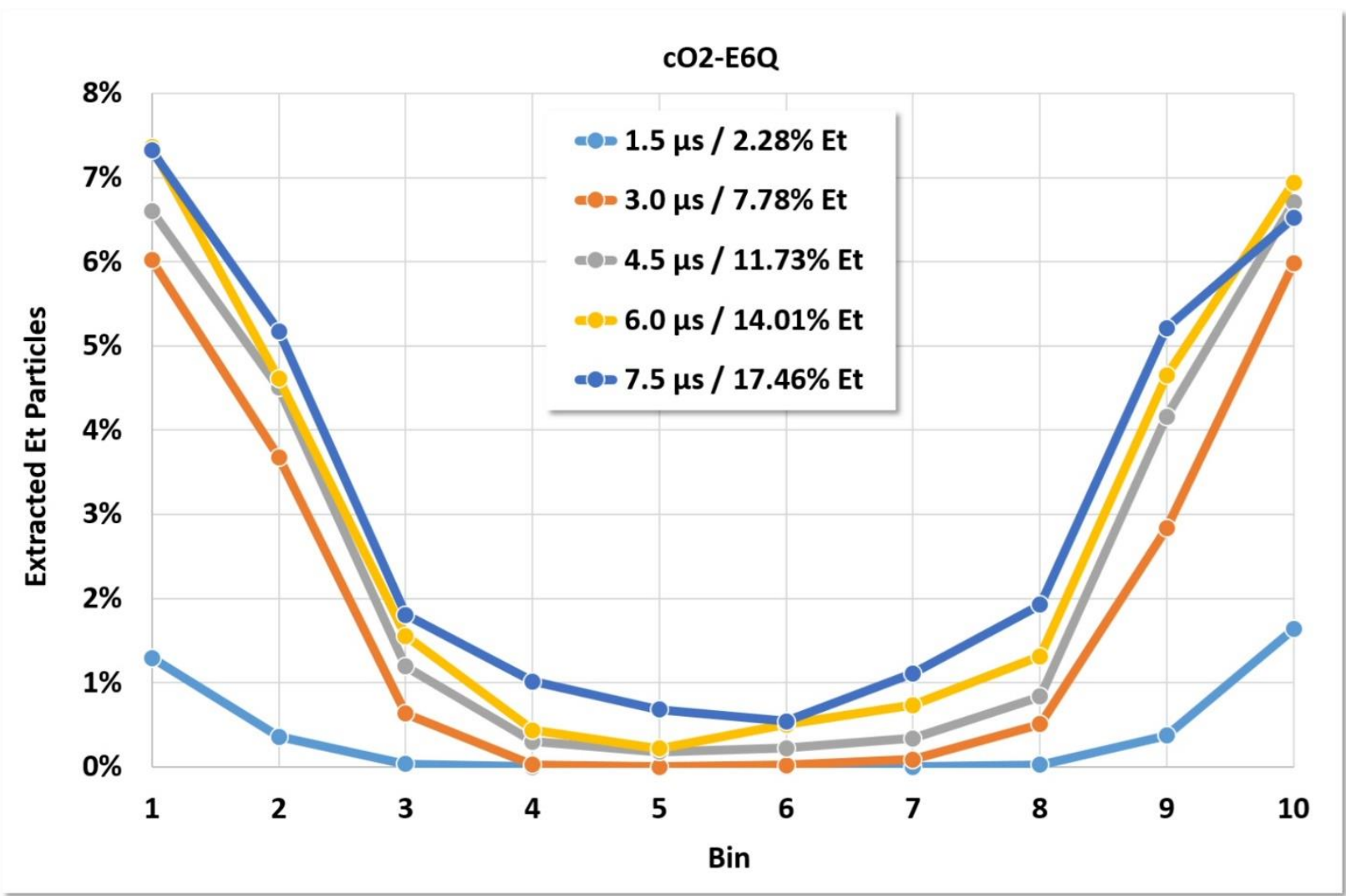

Figure 7. Distribution of extracted Et particles in the cyclotide/water compartment at different simulation times for system $\mathrm{CO} 2-\mathrm{E} 6 \mathrm{Q} /$ NoC-PM. 
The phospholipid extraction into bins 2 to 9 is evaluated 25 times for every $0.3 \mu \mathrm{s}$ over $7.5 \mu \mathrm{s}$. Figure 8 shows the results for 10 simulation runs with different random start geometries for each of three cyclotide/membrane systems (kB1-W19Y-P20S$\mathrm{V} 21 \mathrm{~T} /$ NoC-PM, kB1/NoC-PM and $\mathrm{cO} 2-\mathrm{E} 6 \mathrm{Q} /$ NoC-PM) which span the range from low

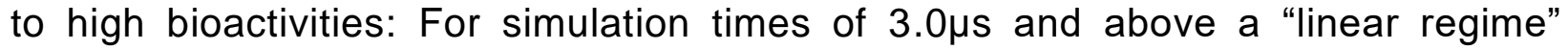
appears that may be approximated by a straight line from the fixed point of origin (no extracted Et particles at simulation start).

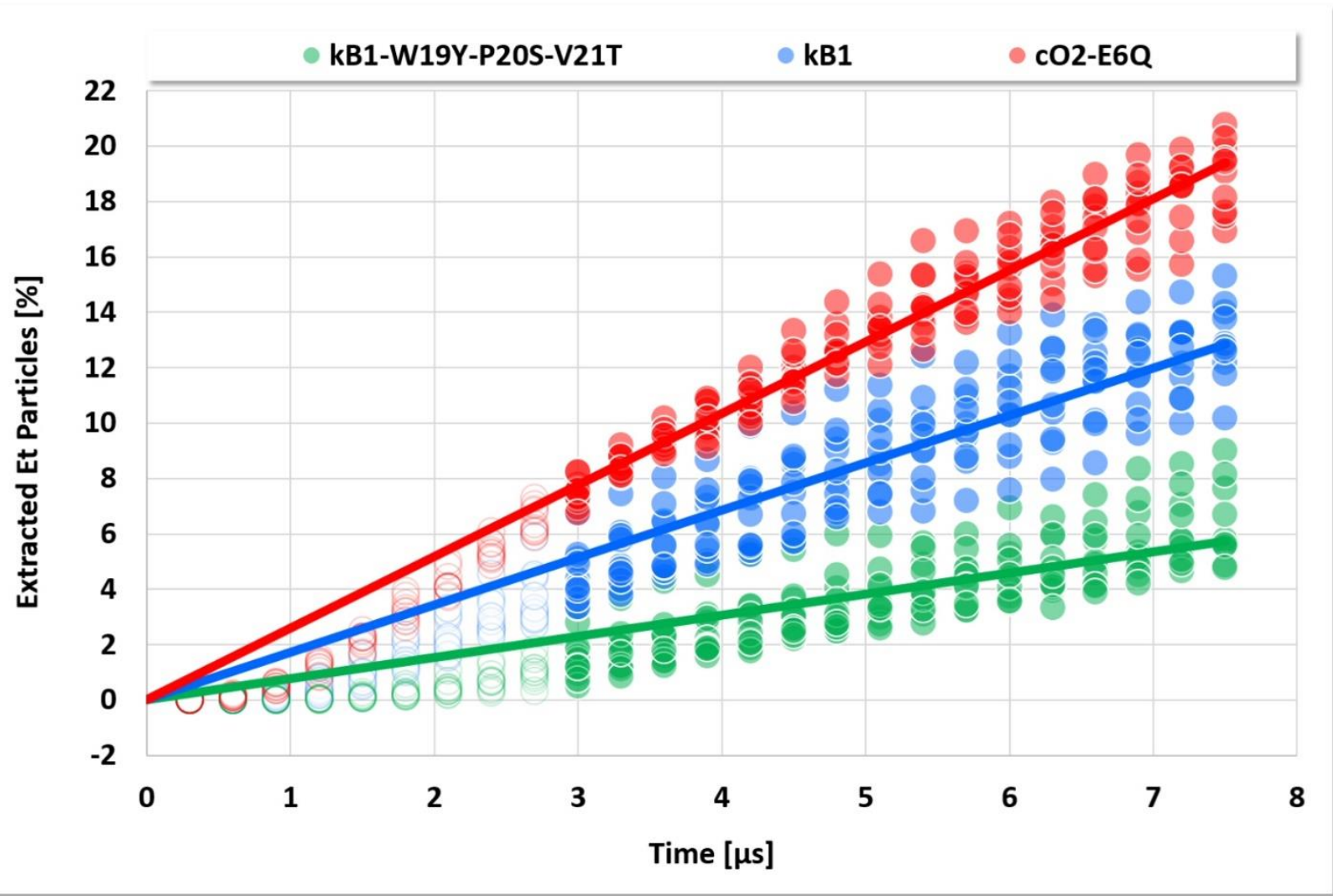

Figure 8. Total inside-bins-2-to-9 extracted-Et percentages evaluated every $0.3 \mu \mathrm{s}$ over $7.5 \mu$ s for 10 simulation runs with different random start geometries for each of the cyclotide/membrane systems kB1-W19Y-P20S-V21T/NoC-PM, kB1/NoC-PM and $\mathrm{CO} 2-\mathrm{E} 6 \mathrm{Q} / \mathrm{NoC}-\mathrm{PM}$. The straight regression lines are fixed at the origin with their slopes being fit to the data for simulation times from 3.0 to $7.5 \mu \mathrm{s}$.

Thus, the total extracted Et percentages (the "areas" under the Et distribution "curves" in figure 7) can be characterized by a single rate constant for membrane disruption (abbreviated $r_{M D}$ ) being the slope of this linear regression line as an 
averaged percentage of extracted Et particles per microsecond: A higher $r_{M D}$ value corresponds to a higher membrane disruption activity with a value of zero indicating no membrane disruption by lipid extraction. Rate constants denoted $r_{M D}(6.0)$ refer to an evaluation of data between 3.0 and $6.0 \mu$ s whereas $r_{M D}(7.5)$ rate constants are derived from data between 3.0 and $7.5 \mu$ s.

From the 10-fold repeated simulation runs with different random start geometries the standard error of a single-run $r_{M D}$ value can be estimated to be about 0.2 units so that for a 10 -fold repetition average the error drops below 0.1 units. The sketched evaluation procedure itself (with $25 \times 10=250$ simulation box distributions) - denoted full evaluation - can be considerably reduced without any significant loss by just evaluating distinct simulation times at $3.0,4.5,6.0$ and $7.5 \mu$ s (with $4 \times 10=40$ simulation box distributions) for $r_{M D}(7.5)$ rate constants or 3.0, 4.5 and $6.0 \mu$ s (with $3 \times 10=30$ simulation box distributions) for $r_{M D}(6.0)$ rate constants respectively denoted reduced evaluation. In addition, the resulting $r_{M D}(6.0)$ and $r_{M D}(7.5)$ values do not exhibit significant differences for the cyclotide/membrane systems studied. For minimal effort, a 4-fold repetition with different random start geometries and reduced $r_{M D}(6.0)$ rate constant evaluation (with $4 \times 3=12$ simulation box distributions) may be chosen which leads to an error of the resulting averaged $r_{M D}$ rate constant of about 0.1 units. If the detected spread of the single-run $r_{M D}$ values is larger for a specific cyclotide/membrane system (as it is the case for $k B 1 /$ NoC-invPM) the number of repetitions with different random start geometries can be increased accordingly to arrive at an acceptable error for the average. To ease comprehension the bioactivity of $r_{M D}$ rate constants between 0 and 1 will be characterized as "low", values between 1 and 2 as "medium" and values above 2 as "high". Note, that a further "standardization" could be introduced by defining a relative bioactivity $r_{\text {rel }}=$

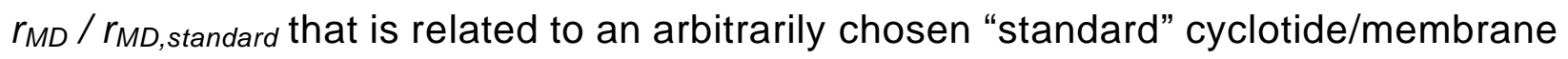
system. For all cyclotide/membrane systems of this study the averaged $r_{M D}$ rate constants with their errors and their type of evaluation are collected in table 1: The following discussions refer to this table. 
Table 1. Rate constants for membrane disruption by lipid extraction

\begin{tabular}{|c|c|c|c|c|}
\hline \multirow[b]{2}{*}{ Cyclotide / Membrane } & \multicolumn{2}{|c|}{ full evaluation } & \multicolumn{2}{|c|}{ reduced evaluation } \\
\hline & $r_{M D}(6.0)$ & $r_{M D}(7.5)$ & $r_{M D}(6.0)$ & $r_{M D}(7.5)$ \\
\hline $\mathrm{kB1} / \mathrm{50C-PM}{ }^{[\mathrm{b}]}$ & & & $1.41 \pm 0.07$ & \\
\hline kB1 / NoC-PM [a] & $1.68 \pm 0.07$ & $1.71 \pm 0.06$ & $1.69 \pm 0.07$ & $1.71 \pm 0.05$ \\
\hline kB1 / NoC-DMPC-M ${ }^{\text {[a] }}$ & $2.01 \pm 0.07$ & $2.10 \pm 0.06$ & $2.04 \pm 0.07$ & $2.10 \pm 0.05$ \\
\hline kB1 / NoC-invPM [c] & & & $2.93 \pm 0.26$ & \\
\hline kB1 / NoC-DOPE-M ${ }^{[b]}$ & & & $3.18 \pm 0.07$ & \\
\hline kB1-W19Y / 50C-PM ${ }^{[b]}$ & & & $0.91 \pm 0.15$ & \\
\hline kB1-W19Y / NoC-PM [b] & & & $1.26 \pm 0.10$ & \\
\hline kB1-W19Y-P20S-V21T / 50C-PM [b] & & & $0.24 \pm 0.04$ & \\
\hline kB1-W19Y-P20S-V21T / NoC-PM [a] & $0.66 \pm 0.04$ & $0.72 \pm 0.04$ & $0.66 \pm 0.04$ & $0.73 \pm 0.04$ \\
\hline kB1-W19Y-P20S-V21T-L27T-P28S-V29T / NoC-PM ${ }^{[b]}$ & & & $0.02 \pm 0.01$ & \\
\hline kB1-W19K / 50C-PM ${ }^{[b]}$ & & & $0.98 \pm 0.08$ & \\
\hline kB1-W19K / NoC-PM ${ }^{[b]}$ & & & $1.46 \pm 0.08$ & \\
\hline kB1-T16K-N25K / 50C-PM ${ }^{[b]}$ & & & $2.08 \pm 0.07$ & \\
\hline KB1-T16K-N25K / NoC-PM ${ }^{[b]}$ & & & $2.53 \pm 0.05$ & \\
\hline $\mathrm{cO} 2 / 50 \mathrm{C}-\mathrm{PM}{ }^{[\mathrm{b}]}$ & & & $1.52 \pm 0.05$ & \\
\hline $\mathrm{cO} 2 / \mathrm{NoC}-\mathrm{PM}{ }^{[\mathrm{b}]}$ & & & $2.26 \pm 0.10$ & \\
\hline cO2 / NoC-DMPC-M ${ }^{[a]}$ & $2.20 \pm 0.07$ & $2.19 \pm 0.06$ & $2.20 \pm 0.07$ & $2.17 \pm 0.06$ \\
\hline cO2 / NoC-invPM ${ }^{[b]}$ & & & $3.24 \pm 0.07$ & \\
\hline cO2 / NoC-DOPE-M ${ }^{[b]}$ & & & $3.46 \pm 0.02$ & \\
\hline cO2-W10Y-I11T-P12S / NoC-PM ${ }^{[b]}$ & & & $1.79 \pm 0.05$ & \\
\hline c02-W10Y-I11T-P12S-I14T-A17S-I18T / NoC-PM [b] & & & $1.36 \pm 0.07$ & \\
\hline CO2-K23Q-K25Q-R29W / NoC-PM [b] & & & $1.89 \pm 0.09$ & \\
\hline cO2-E6Q / 50C-PM ${ }^{[b]}$ & & & $1.85 \pm 0.04$ & \\
\hline cO2-E6Q / NoC-PM ${ }^{[a]}$ & $2.62 \pm 0.05$ & $2.59 \pm 0.05$ & $2.59 \pm 0.05$ & $2.56 \pm 0.05$ \\
\hline kB1-W19Y-P20S-V21T (875) / cO2-E6Q (125) / NoC-PM [b] & & & $1.10 \pm 0.06$ & \\
\hline kB1-W19Y-P20S-V21T (750) / cO2-E6Q (250) / NoC-PM [a] & $1.19 \pm 0.06$ & $1.25 \pm 0.06$ & $1.20 \pm 0.06$ & $1.27 \pm 0.06$ \\
\hline kB1-W19Y-P20S-V21T (625) / cO2-E6Q (375) / NoC-PM ${ }^{[b]}$ & & & $1.56 \pm 0.10$ & \\
\hline kB1-W19Y-P20S-V21T (500) / cO2-E6Q (500) / NoC-PM [a] & $1.80 \pm 0.03$ & $1.83 \pm 0.04$ & $1.79 \pm 0.04$ & $1.81 \pm 0.04$ \\
\hline kB1-W19Y-P20S-V21T (375) / cO2-E6Q (625) / NoC-PM ${ }^{[b]}$ & & & $1.97 \pm 0.05$ & \\
\hline kB1-W19Y-P20S-V21T (250) / cO2-E6Q (750) / NoC-PM ${ }^{[a]}$ & $2.17 \pm 0.05$ & $2.18 \pm 0.04$ & $2.17 \pm 0.05$ & $2.16 \pm 0.04$ \\
\hline kB1-W19Y-P20S-V21T (125) / cO2-E6Q (875) / NoC-PM ${ }^{[b]}$ & & & $2.48 \pm 0.07$ & \\
\hline
\end{tabular}

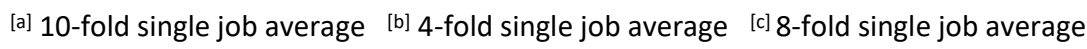




\section{Membrane-dependent bioactivity}

The interaction of kB1 with 50C-PM, NoC-PM, NoC-DMPC-M and NoC-DOPE-M exhibits an increasing membrane lipid extraction from medium to very high activity. The same trend is found for $\mathrm{CO} 2$ (where the $\mathrm{CO} 2$ interaction with NoC-PM and NoCDMPC-M is of comparable activity whereas the kB1 interaction with NoC-DMPC-M is higher than with NoC-PM). The $\mathrm{CO} 2$ activities for all four membrane types studied are above those for kB1. These findings are in agreement with the experimental study in [17] that characterizes $\mathrm{cO} 2$ as the "most potent cytotoxic and antimicrobial cyclotide" in comparison to the Möbius cyclotides kB1 and Kalata B2 as well as in [15] where $\mathrm{cO} 2$ is characterized as the "the most active cyclotide" compared to the Möbius ones.

The decreased activity of $\mathrm{kB} 1$ and $\mathrm{CO} 2$ with $50 \mathrm{C}-\mathrm{PM}$ in comparison to NoC-PM can be traced to a difference between phospholipid and cholesterol extraction: Whereas the phospholipids of a 50C-PM are extracted in a comparable extent to NoC-PM, the cholesterol extraction is significantly reduced. Thus, the extraction of phospholipids is distinctly preferred. The activity decreasing effect of cholesterol is also found for the low activity mutant kB1-W19Y-P20S-V21T, the medium activity mutant kB1-W19K as well as the super-active mutants kB1-T16K-N25K and cO2E6Q. A decrease of cyclotide membrane disrupting activity due to cholesterol is also experimentally observed [17] as well as detected by coarse-grained MD simulations [38].

The influence of the membrane composition on the cyclotide activity becomes most obvious for the kB1 and $\mathrm{CO} 2$ interaction with NoC-DOPE-M which leads to a considerable "activity jump" for both cyclotides in comparison to the other membrane types. The fact that the phospholipid membrane composition significantly influences the disrupting activity with a trend towards higher activities for PE-rich membranes is also experimentally shown in [19, 21-23, 25, 29, 31].

Since the phospholipid composition of the inner and outer leaflet of a plasma membrane differs, an inversion of the plasma membrane on the $\mathrm{kB} 1$ and $\mathrm{cO} 2$ activity was studied in addition so that the cyclotides primarily interact with the inner membrane leaflets instead of the outer ones. For cholesterol-free inverted plasma 
membranes (NoC-invPM) kB1 and $\mathrm{cO} 2$ exhibit a considerably increased activity compared to the non-inverted ones - even close to pure NoC-DOPE-M: The increased activity with the inner membrane leaflet may be partly attributed to the higher (77.5 to 22.5) DOPE concentration of the inner leaflet which is again in agreement with the detected increased membrane disrupting activity with PE-rich membranes.

\section{"Hydrophobic patch" mutants}

The study of cyclotide mutants with specific amino acid replacements may indicate the importance of single or multiple amino acids for the lipid extraction process. Mutant kB1-W19Y consists of a single amino acid replacement in the "hydrophobic patch" (compare figure 3) where amino acid tryptophan (W) at position 19 is replaced by the more polar amino acid tyrosine $(\mathrm{Y})$ : This single spot mutation already leads to a decreased membrane disruption activity for NoC-PM and 50C-PM in comparison with native kB1. Triple mutant kB1-W19Y-P20S-V21T (with half of the amino acids in the "hydrophobic patch" being replaced by more polar/hydrophilic ones) shows a further decreased activity for NoC-PM and 50C-PM and the final "fully mutated" kB1W19Y-P20S-V21T-L27T-P28S-V29T mutant (where all six "hydrophobic patch" amino acids are correspondingly replaced so that the "hydrophobic patch" turns into a "hydrophilic patch") does no longer show any significant lipid extraction with NoCPM.

The animation at [87] and figure 9 demonstrate the activity decrease due to "hydrophobic patch" mutations: The increasingly polar/hydrophilic transformation of "hydrophobic patch" amino acids still allows for cyclotide oligomerization but leads to a decreased tendency to aggregate into networks: This in turn diminishes the "hydrophobic vicinities" so that the collective lipid extraction abilities are more and more reduced. 


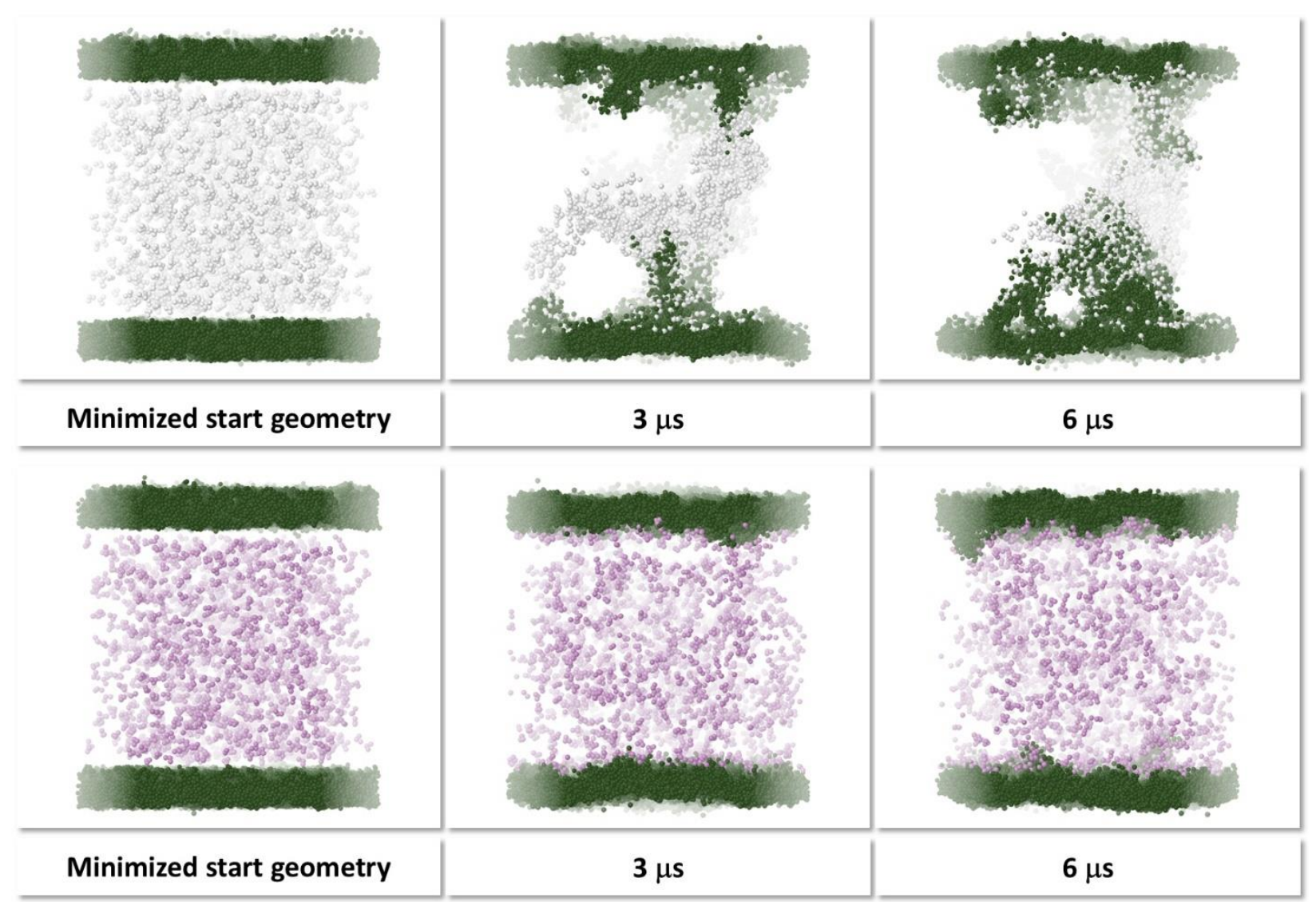

Figure 9. Interaction snapshots of kB1 (upper row) and the "hydrophilic patch" mutant kB1-W19Y-P20S-V21T-L27T-P28S-V29T (lower row) with NoC-PM at different simulation times for the same simulation box cross-section view. The backbone particles of the kB1 "hydrophobic patch" are colored in white (upper row), the mutated backbone particles of the "hydrophobic patch" in plum (lower row). All other cyclotide backbone and sidechain particles are omitted. Only "hydrophobic" phospholipid particles are shown in olive. All water particles are omitted.

Replacement of the six Cycloviolacin O2 "hydrophobic patch" amino acids (compare figure 3 ) with more polar/hydrophilic ones analog to kB1 leads to a corresponding decrease in bioactivity for NoC-PM: Triply mutated CO2-W10Y-I11TP12S exhibits a significant activity decrease and the activity of the "fully mutated hydrophilic patch" mutant cO2-W10Y-I11T-P12S-I14T-A17S-I18T becomes even smaller although being still in the medium activity region (unlike the corresponding completely inactive mutant kB1-W19Y-P20S-V21T-L27T-P28S-V29T).

The importance of the "hydrophobic patch" surface region for a cyclotide's bioactivity has also been emphasized by experimental studies $[8,14,19,34]$ where 
the activity decrease due to polar/hydrophilic amino acid replacements/modifications is in agreement with experimental findings.

\section{Super-mutants and charge state}

Experimental results in [5, 15, 17-19] indicate that replacements of or with charged amino acids may cause considerable changes in activity including super-active mutants but without any simple patterns. The same holds true for the influence of the total net charge. These findings are supported by corresponding simulations with differently charged cyclotide mutants:

Mutant kB1-W19K (total net charge +1 ) consists of a single amino acid replacement in the "hydrophobic patch" region (compare figure 3) where hydrophobic amino acid tryptophan (W) at position 19 is replaced with the positively charged amino acid lysine (K). For interaction with NoC-PM this leads to a slightly decreased membrane disrupting activity and for 50C-PM to a significant decrease compared with native kB1 (total net charge 0 ) but the activity is still in or at the edge of the medium region. In [19] mutant kB1-W19K (denoted "W23K" in this publication) was experimentally studied in different membrane systems and characterized to be "inactive".

In mutant kB1-T16K-N25K (total net charge +2 ) two uncharged amino acids are replaced with positively charged lysine $(\mathrm{K})$ : Compared to native $\mathrm{kB} 1$ these mutations lead to considerably increased membrane disruptions for 50C-PM (already in the high activity region) and NoC-PM (well in the high activity region). The experimental study of this mutant with different membrane systems leads to a "more potent" characterization in comparison with kB1 [19] (where this mutant is denoted T20K/N29K).

In mutant $\mathrm{cO} 2-\mathrm{E} 6 \mathrm{Q}$ (total net charge +3 ) the negatively charged glutamic acid $(E)$ at position 6 is replaced with the uncharged amino acid glutamine $(Q)$. Together with kB1-T16K-N25K this mutant exhibits the highest membrane disrupting activity of all studied cyclotides for 50C-PM and NoC-PM well above native cO2 (total net charge $+2)$ which agrees with experimental findings in [17] for the interaction of DOPC/DOPA liposomes with a chemically modified $\mathrm{cO} 2$ derivate where the glutamic 
acid was esterified by acetyl chloride - while the opposite is reported for other membrane types $[5,15,18]$.

Mutant cO2-K23Q-K25Q-R29W (total net charge -1) has all positively charged amino acids replaced with uncharged alternatives. For NoC-PM this mutant performs below the activity of native $\mathrm{cO} 2$ which agrees with experimental results in [5].

\section{Additivity of cyclotide activity}

Figure 10 demonstrates the influence of kB1-W19Y-P20S-V21T/cO2-E6Q cyclotide mixtures with NoC-PM on the membrane disrupting activity, indicating a linear additivity without significant under- or over-additive effects which is in agreement with experimental findings in [18]. Note, that an analysis of cyclotide mixtures may also be utilized for comparative assessments of cyclotide/membrane systems with similar bioactivity with the sign of the regression line slope as a threshold for attributing lower (negative sign) or higher activity (positive sign) to the cyclotide with an increasing fraction. 


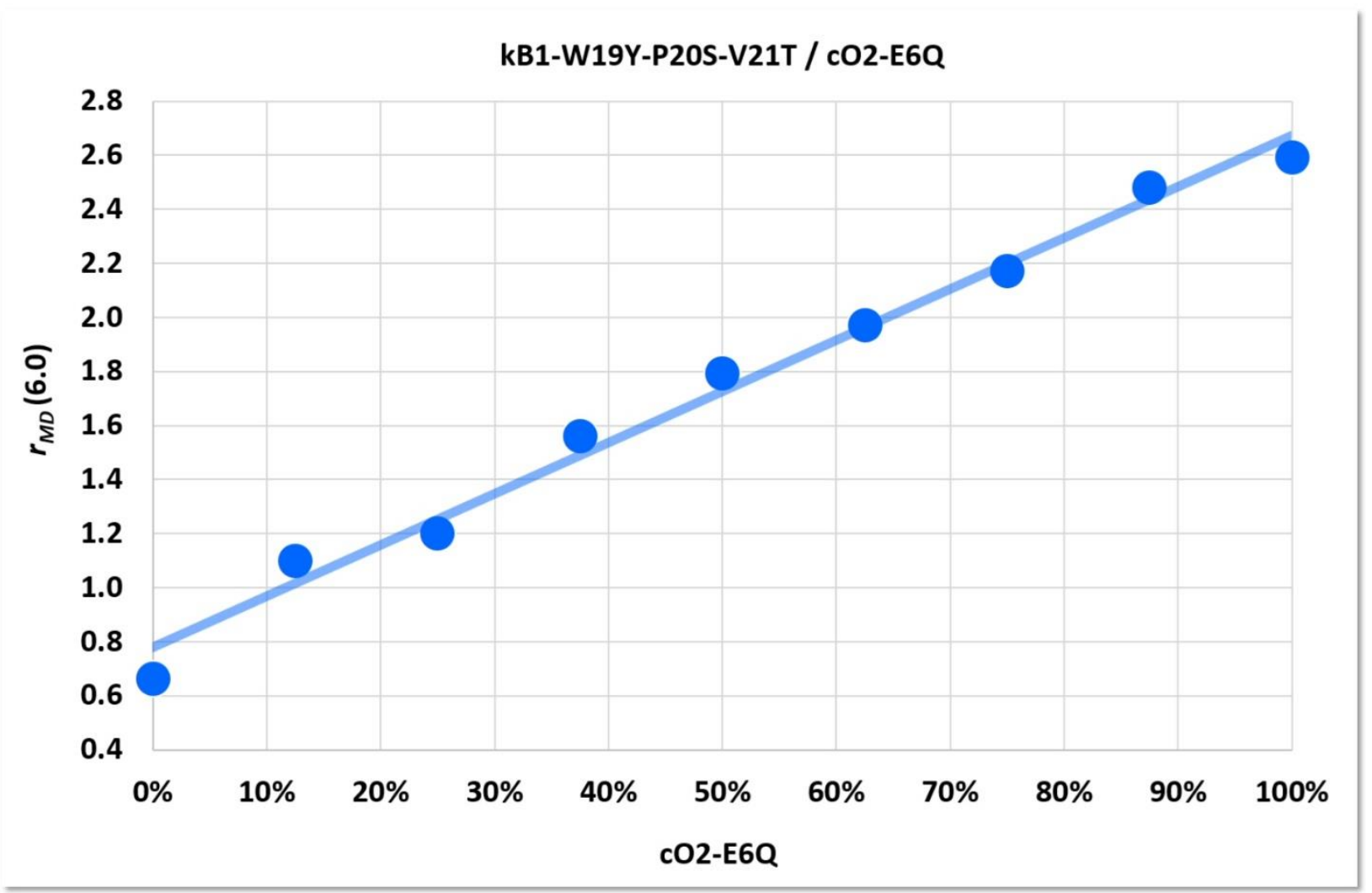

Figure 10. Rate constants $r_{M D}(6.0)$ for membrane disruption of different kB1-W19YP20S-V21T/ cO2-E6Q mixtures with NoC-PM. The coefficient of determination $\left(R^{2}\right)$ for the sketched linear regression line is above 0.98 .

\section{Pore formation}

As a side note to the contentious discussion about the possible role of cyclotideinduced pore formation for the membrane disruption process $[3,5-7,9,11,13,14$, $16,17,19-24,26,29,31,35-38$ ] it should be mentioned that all simulation models of this work do not exhibit any stable membrane pores due to cyclotide interaction over the studied $7.5 \mu$ s time span.

\section{Conclusions}

In this work an attempt is made to quantify cyclotide-induced bilayer membrane disruption by lipid extraction to obtain characteristic system-specific rate constants for a "sandwich" interaction model at the microsecond timescale. The mesoscopic 
simulation results agree with experimentally observed trends with the virtue of being compressible into simple numbers that allow for comparative assessments and structure-activity relationship (SAR) studies. Thus, the proposed method may support a deeper exploration of the complex cyclotide/membrane bioactivity landscape, see figure 11 .

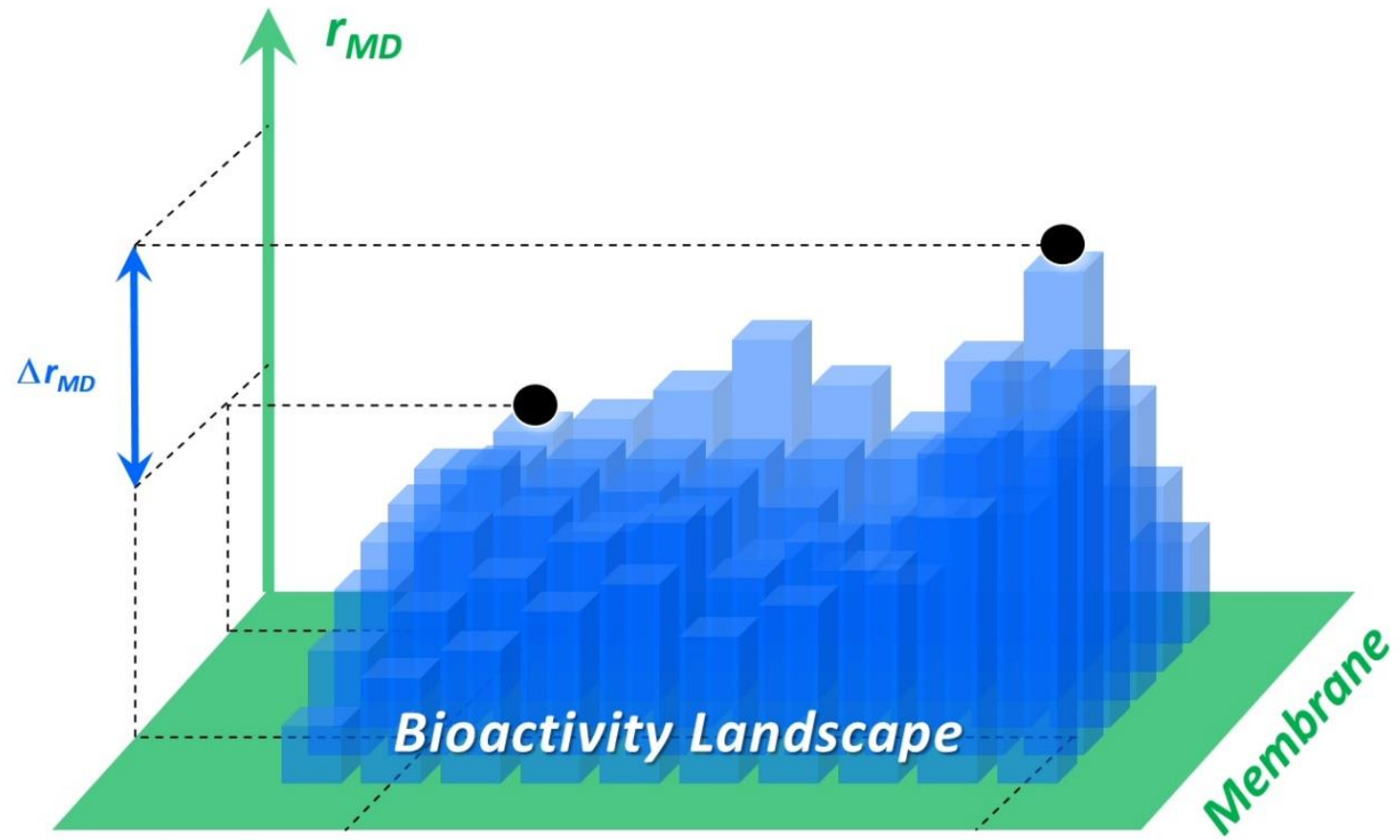

\section{Cyclotide}

Figure 11. Schematic view of the cyclotide/membrane bioactivity landscape suggested by this work where each bar represents the membrane disruption activity of a specific cyclotide/membrane system and each row (column) of bars corresponds to a specific membrane (cyclotide).

In general, any approximate approach is questionable on three successive levels from the abstract to the concrete: (I) Is the chosen level of theory capable of describing the phenomena in question? (II) Is the concrete model setup in combination with its parameter settings adequate? (III) Can the model successfully be applied to the specific systems under investigation? "Coarse grained" mesoscopic simulation is a method of choice for studying emergent collective phenomena in large 
chemical systems due to non-bonded interactions at the microsecond scale correspondingly more elaborate atomistic approaches would increase the computational effort by orders of magnitude. The "sandwich" interaction model with the provided particle data and the chosen molecular fragmentation is able to show cyclotide-induced lipid extraction. Finally, the quantitatively evaluated bioactivities for the studied cyclotide/membrane systems reflect experimental results and trends. In summary, the application results of the mesoscopic "sandwich" model indicate positive answers to the raised questions so that the model is likely to comprise relevant "elements of reality". Nevertheless, the approach is open for substantial improvements.

Additional open biomolecular particle sets and alternative fragmentation schemes are in need for comparative evaluations (to our knowledge none are openly available at the moment). Besides the proposed "sandwich" interaction model various modified interaction setups should be explored and comparatively studied - not least to shed light on principle limits: Whenever a disruptive cyclotide/membrane interaction is driven by specific atomic interactions that cannot be adequately abstracted in mesoscopic particle-particle interaction averages, the mesoscopic level becomes prohibitive. The combined efforts of improved simulation setups and corresponding experimental studies may eventually lead to proper assessments.

An attractive but often undervalued feature of mesoscopic approaches with open rich-client end-user systems is their fast performance ("one-day effort") in combination with a comparatively low barrier to entry ("familiarization within hours", compare the animated tutorial for setup and evaluation of the outlined "sandwich" interaction model with MFsim [55, 56]). In consequence, end-user accessibility allows for simulation studies and lab experiments to be executed in a concurrent manner by the experimental scientists themselves (there are no extravagant hardware devices nor programming skills required) so that the simulation part could be advantageously integrated into the experimental research workflow. 


\section{Appendix}

Overview and links to the animations created for this work:

- Tutorial MFsim - Cyclotide-membrane sandwich interaction model [56]

- Plasma membrane cholesterol mobility [69]

- $\mathrm{kB} 1$ and $\mathrm{cO} 2$ backbone flexibility [82]

- $\mathrm{kB1}$ membrane interaction [83]

- Quantitative cyclotide-membrane interaction analysis [86]

- $\quad$ kB1 "hydrophobic patch" mutants membrane interaction [87]

\section{Declarations}

Authors' contributions: KvdB designed and performed the simulations. LSK supported the evaluation and documentation process. ME, HK and AZ lead the project progress. All authors read and approved the final manuscript.

Acknowledgements: The support of CAM-D Technologies GmbH and GNWI Gesellschaft für naturwissenschaftliche Informatik mbH is gratefully acknowledged. 


\section{References}

[1] Craik DJ, Daly NL, Bond T, Waine C (1999) Plant Cyclotides: A Unique Family of Cyclic and Knotted Proteins that Defines the Cyclic Cystine Knot Structural Motif. Journal of Molecular Biology 294:1327-1336

[2] de Veer J, Kan MW, Craik DJ (2019) Cyclotides: From Structure to Function. Chemical Reviews 119:12375-12421

[3] Lindholm P, Göransson U, Johansson S, Claeson P, Gullbo J, Larsson R, Bohlin L, Backlund A (2002) Cyclotides: A Novel Type of Cytotoxic Agents. Molecular Cancer Therapeutics 1:365-369

[4] Barry DG, Daly NL, Clark RJ, Sando L, Craik DJ (2003) Linearization of a Naturally Occurring Circular Protein Maintains Structure but Eliminates Hemolytic Activity. Biochemistry 42:6688-6695

[5] Herrmann A, Svangård E, Claeson P, Gullbo J, Bohlin L, Göransson U (2006) Key role of glutamic acid for the cytotoxic activity of the cyclotide cycloviolacin O2. Cellular and Molecular Life Sciences 63:235-245

[6] Shenkarev ZO, Nadezhdin KD, Sobol VA, Sobol AG, Skjeldal L, Arseniev AS (2006) Conformation and mode of membrane interaction in cyclotides. FEBS Journal 273:2658-2672

[7] Svangård E, Burman R, Gunasekera S, Lövborg H, Gullbo J, Göransson U (2007) Mechanism of Action of Cytotoxic Cyclotides: Cycloviolacin O2 Disrupts Lipid Membranes. Journal of Natural Products 70(4):643-647

[8] Simonsen SM, Sando L, Rosengren KJ, Wang CK, Colgrave ML, Daly NL, Craik DJ (2008) Alanine Scanning Mutagenesis of the Prototypic Cyclotide Reveals a Cluster of Residues Essential for Bioactivity. Journal of Biological Chemistry 283:9805-9813

[9] Wang CK, Hu SH, Martin JL, Sjogren T, Hajdu J, Bohlin L, Claeson P, Göransson U, Rosengren KJ, Tang J, Tan NH, Craik DJ (2009) Combined Xray and NMR Analysis of the Stability of the Cyclotide Cystine Knot Fold That Underpins Its Insecticidal Activity and Potential Use as a Drug Scaffold. Journal of Biological Chemistry 284(16):10672-10683 
[10] Wang CK, Colgrave ML, Ireland DC, Kaas Q, Craik DJ (2009) Despite a Conserved Cystine Knot Motif, Different Cyclotides Have Different Membrane Binding Modes. Biophysical Journal 97:1471-1481

[11] Huang YH, Colgrave ML, Daly NL, Keleshian A, Martinac B, Craik DJ (2009) The Biological Activity of the Prototypic Cyclotide Kalata B1 Is Modulated by the Formation of Multimeric Pores. Journal of Biological Chemistry 284(31):20699-20707

[12] Göransson U, Herrmann A, Burman R, Haugaard-Jönsson LM, Rosengren KJ (2009) The Conserved Glu in the Cyclotide Cycloviolacin O2 Has a Key Structural Role. ChemBioChem 10:2354-2360

[13] Gerlach SL, Rathinakumar R, Chakravarty G, Göransson U, Wimley WC, Darwin SP, Mondal D (2010) Anticancer and Chemosensitizing Abilities of Cycloviolacin O2 from Viola odorata and Psyle Cyclotides from Psychotria leptothyrsa. Peptide Science 94(5):617-625

[14] Huang YH, Colgrave ML, Clark RJ, Kotze AC, Craik DJ (2010) Lysine-scanning Mutagenesis Reveals an Amendable Face of the Cyclotide Kalata B1 for the Optimization of Nematocidal Activity. Journal of Biological Chemistry 285:10797-10805

[15] Pränting M, Lööv C, Burman R, Göransson U, Andersson DI (2010) The cyclotide cycloviolacin $\mathrm{O} 2$ from Viola odorata has potent bactericidal activity against Gram-negative bacteria. Journal of Antimicrobial Chemotherapy 65:1964-1971

[16] Wimley WC (2010) Describing the mechanism of antimicrobial peptide action with the interfacial activity model. Chemical Biology 5(10):905-917

[17] Burman R, Strömstedt AA, Malmsten M, Göransson U (2011), Cyclotidemembrane interactions: Defining factors of membrane binding, depletion and disruption. Biochimica et Biophysica Acta 1808:2665-2673

[18] Burman R, Herrmann A, Tran R, Kivelä JE, Lomize A, Gullbo J, Göransson U (2011) Cytotoxic potency of small macrocyclic knot proteins: Structure-activity and mechanistic studies of native and chemically modified cyclotides. Organic and Biomolecular Chemistry 9:4306-4314 
[19] Henriques ST, Huang YH, Rosengren KJ, Franquelim HG, Carvalho FA, Johnson A, Sonza S, TachedjianG, Castanho MARB, Daly NL, Craik DJ (2011) Decoding the Membrane Activity of the Cyclotide Kalata B1. Journal of Biological Chemistry 286(27):24231-24241

[20] Wimley WC, Hristova K (2011) Antimicrobial peptides: successes, challenges and unanswered questions. Journal of Membrane Biology 239:27-34

[21] Wang CK, Wacklin HP, Craik DJ (2012) Cyclotides Insert into Lipid Bilayers to Form Membrane Pores and Destabilize the Membrane through Hydrophobic and Phosphoethanolamine-specific Interactions. Journal of Biological Chemistry 287(52):43884-43898

[22] Henriques ST, Craik DJ (2012) Importance of the Cell Membrane on the Mechanism of Action of Cyclotides. Chemical Biology 7:626-636

[23] Henriques ST, Huang YH, Castanho MARB, Bagatolli LA, Sonza S, Tachedjian G, Daly NL, Craik DJ (2012) Phosphatidylethanolamine Binding is a Conserved Feature of Cyclotide-membrane Interactions. Journal of Biological Chemistry 287:33629-33643

[24] Göransson U, Burman R, Gunasekera S, Strömstedt AA, Rosengren KJ (2012) Circular Proteins from Plants and Fungi. Journal of Biological Chemistry 287(32):27001-27006

[25] Henriques ST, Huang YH, Chaousis S, Wang CK, Craik DJ (2014) Anticancer and Toxic Properties of Cyclotides are Dependent on Phosphatidylethanolamine Phospholipid Targeting. ChemBioChem 15:19561965

[26] Burman R, Gunasekera S, Strömstedt A, Göransson U (2014) Chemistry and Biology of Cyclotides: Circular Plant Peptides Outside the Box. Journal of Natural Products 77:724-736

[27] Henriques ST, Huang YH, Chaousis S, Sani MA, Poth AG, Separovic F, Craik DJ (2015) The Prototypic Cyclotide Kalata B1 Has a Unique Mechanism of Entering Cells. Chemistry \& Biology 22:1087-1097 
[28] Weidmann J, Craik DJ (2016) Discovery, structure, function, and applications of cyclotides: circular proteins from plants. Journal of Experimental Botany 67(16):4801-4812

[29] Cranfield CG, Henriques ST, Martinac B, Duckworth P, Craik DJ, Cornell B (2017) Kalata B1 and Kalata B2 Have a Surfactant-Like Activity in Phosphatidylethanolomine-Containing Lipid Membranes. Langmuir 33:6630-6637

[30] Ghani HA, Henriques ST, Huang YH, Swedberg JE, Schroeder CI, Craik DJ (2017) Structural and functional characterization of chimeric cyclotides from the Möbius and trypsin inhibitor subfamilies. Peptide Science 108:e22927

[31] Grage SL, Sani MA, Cheneval O, Henriques ST, Schalck C, Heinzmann R, Mylne JS, Mykhailiuk PK, Afonin S, Komarov IV, Separovic F, Craik DJ, Ulrich1 AS (2017) Orientation and Location of the Cyclotide Kalata B1 in Lipid Bilayers Revealed by Solid-State NMR. Biophysical Journal 112:630-642

[32] Henriques ST and Craik DJ (2017) Cyclotide Structure and Function: The Role of Membrane Binding and Permeation. Biochemistry 56:669-682

[33] Henriques ST, Peacock H, Benfield AH, Wang CK, Craik DJ (2019) Is the Mirror Image a True Reflection? Intrinsic Membrane Chirality Modulates Peptide Binding. Journal of the American Chemical Society 141:20460-20469

[34] Du Q, Chan LY, Gilding EK, Henriques ST, Condon ND, Ravipati AS, Kaas Q, Huang YH, and Craik DJ (2020) Discovery and mechanistic studies of cytotoxic cyclotides from the medicinal herb Hybanthus enneaspermus. Journal of Biological Chemistry 295(32):10911-10925

[35] Nawae W, Hannongbua S, Ruengjitchatchawalya M (2014) Defining the Membrane Disruption Mechanism of Kalata B1 via Coarse-grained Molecular Dynamics Simulations. Scientific Reports 4:3933

[36] Nawae W, Hannongbua S, Ruengjitchatchawalya M (2014) Dynamic Scenario of Membrane Binding Process of Kalata B1. PLoS ONE 9(12):e114473

[37] Truszkowski A, van den Broek K, Kuhn H, Zielesny A, Epple M (2015) Mesoscopic Simulation of Phospholipid Membranes, Peptides, and Proteins 
with Molecular Fragment Dynamics. Journal of Chemical Information and Modeling 55:983-997

[38] Nawae W, Hannongbua S, Ruengjitchatchawalya M (2017) Molecular dynamics exploration of poration and leaking caused by kalata B1 in HIVinfected cell membrane compared to host and HIV membranes. Scientific Reports 7:3638

[39] Hoogerbrugge PJ, Koelman JMVA (1992) Simulating Microscopic Hydrodynamic Phenomena with Dissipative Particle Dynamics. Europhysics Letters 19(3):155-160

[40] Koelman JMVA, Hoogerbrugge PJ (1993) Dynamic Simulations of HardSphere Suspensions Under Steady Shear. Europhysics Letters 21(3):363-368

[41] Espanol P, Warren P (1995) Statistical Mechanics of Dissipative Particle Dynamics. Europhysics Letters 30(4):191-196

[42] Espanol P (1995) Hydrodynamics from dissipative particle dynamics. Physical Review E 52(2):1734-1742.

[43] Groot RD, Warren P (1997) Dissipative particle dynamics: Bridging the gap between atomistic and mesoscopic simulation. Journal of Chemical Physics 107(11):4423-4435

[44] Groot RD, Madden TJ (1998) Dynamic simulation of diblock copolymer microphase separation. Journal of Chemical Physics 105(20):8713-8724

[45] Ryjkina E, Kuhn H, Rehage H, Müller F, Peggau J (2002) Molecular Dynamic Computer Simulations of Phase Behavior of Non-Ionic Surfactants. Angewandte Chemie International Edition 41(6):983-986

[46] Schulz SG, Kuhn H, Schmid G, Mund C, Venzmer J (2004) Phase behavior of amphiphilic polymers: A dissipative particles dynamics study. Colloid and Polymer Science 283:284-290

[47] Truszkowski A, Epple M, Fiethen A, Zielesny A, Kuhn H (2013) Molecular fragment dynamics study on the water-air interface behavior of non-ionic polyoxyethylene alkyl ether surfactants. Journal of Colloid and Interface Science 410:140-145 
[48] Vishnyakov A, Lee M-T, Neimark AV (2013) Prediction of the Critical Micelle Concentration of Nonionic Surfactants by Dissipative Particle Dynamics Simulations. Journal of Physical Chemistry Letters 4:797-802

[49] Truszkowski A, Daniel M, Kuhn H, Neumann S, Steinbeck C, Zielesny A, Epple $M$ (2014) A molecular fragment cheminformatics roadmap for mesoscopic simulation. Journal of Cheminformatics 6:45

[50] van den Broek K, Daniel M, Epple M, Hein JM, Kuhn H, Neumann S, Truszkowski A, Zielesny A (2020) MFsim - an open Java all-in-one rich-client simulation environment for mesoscopic simulation. Journal of Cheminformatics 12:29

[51] van den Broek K, Kuhn H, Zielesny A (2018) Jdpd - an open java simulation kernel for molecular fragment dissipative particle dynamics. Journal of Cheminformatics 10:25

[52] van den Broek K, Daniel M, Epple M, Kuhn H, Schaub J and Zielesny A (2018) SPICES: a particle-based molecular structure line notation and support library for mesoscopic simulation. Journal of Cheminformatics 10:35.

[53] Protein Data Bank. https://www.rcsb.org. Accessed 23 February 2021.

[54] MFsim particle set text file ParticleSet_AA_V03.txt in MFsim_Source/particles subfolder of MFsim repository. https://github.com/zielesny/MFsim. Accessed 23 February 2021.

[55] MFsim - Cyclotide-membrane sandwich interaction model. PDF document in tutorials section. https://github.com/zielesny/MFsim. Accessed 23 February 2021.

[56] MFsim - Cyclotide-membrane sandwich interaction model. MP4 clip. https://whs.sciebo.de/s/QTIhqhQyBR9tSRW. Accessed 23 February 2021.

[57] Subfolder 2021 Cyclotide-membrane interaction study of MFsim repository at https://github.com/zielesny/MFsim. Accessed 23 February 2021.

[58] Shardlow T (2003) SPLITTING FOR DISSIPATIVE PARTICLE DYNAMICS. SIAM Journal on Scientific Computing 24(4):1267-1282

[59] Nikunen P, Karttunen M, Vattulainen I (2003) How would you integrate the equations of motion in dissipative particle dynamics simulations? Computer Physics Communications 153(3):407-423 
[60] Jmol: an open-source browser-based HTML5 viewer and stand-alone Java viewer for chemical structures in 3D. http://jmol.sourceforge.net/. Accessed 23 February 2021.

[61] Daleke DL (2008) Regulation of phospholipid asymmetry in the erythrocyte membrane. Current Opinion in Hematology 15:191-195

[62] van Meer G, Voelker DR, Feigenson GW (2008) Membrane lipids: where they are and how they behave. Nature Reviews Molecular Cell Biology 9:112-124

[63] Yamamoto T (1963) ON THE THICKNESS OF THE UNIT MEMBRANE. Journal of Cell Biology 17(2):413-421

[64] Bar RS, Deamer DW, Cornwell DG (1966) Surface Area of Human Erythrocyte Lipids: Reinvestigation of Experiments on Plasma Membrane. Science 153(3739):1010-1012

[65] Engelman DM (1969) Surface Area per Lipid Molecule in the Intact Membrane of the Human Red Cell. Nature 223:1279-1280

[66] Kiselev MA, Zemlyanaya EV, Aswal VK, Neubert RHH (2006) What can we learn about the lipid vesicle structure from the small-angle neutron scattering experiment? European Biophysics Journal 35:477-493

[67] Kučerka N, Nieh MP, Katsaras J (2011) Fluid phase lipid areas and bilayer thicknesses of commonly used phosphatidylcholines as a function of temperature. Biochimica et Biophysica Acta 1808:2761-2771

[68] Ingólfsson HI, Melo MN, van Eerden FJ, Arnarez C, Lopez CA, Wassenaar TA, Periole X, de Vries AH, Peter Tieleman D, Marrink SJ (2014) Lipid Organization of the Plasma Membrane. Journal of the American Chemical Society 136:14554-14559

[69] Plasma membrane cholesterol mobility. MP4 clip. https://whs.sciebo.de/s/BphC3r3fhcXc3EK. Accessed 23 February 2021.

[70] Lange Y, Dolde J, Steck TL (1981) The Rate of Transmembrane Movement of Cholesterol in the Human Erythrocyte. Journal of Biological Chemistry 256(11):5321-5323 
[71] Hamilton JA (2003) Fast flip-flop of cholesterol and fatty acids in membranes: implications for membrane transport proteins. Current Opinion in Lipidology 14:263-271

[72] Zaccai G, Blasie JK, Schoenborn BP (1975) Neutron Diffraction Studies on the Location of Water in Lecithin Bilayer Model Membranes. Proceedings of the National Academy of Sciences 72(1):376-380

[73] Hoekstra D (1982) Role of Lipid Phase Separations and Membrane Hydration in Phospholipid Vesicle Fusion. Biochemistry 21:2833-2840

[74] Lewis B, Engelman DM (1983) Lipid Bilayer Thickness Varies Linearly with Acyl Chain Length in Fluid Phosphatidylcholine Vesicles. Journal of Molecular Biology 166:211-217

[75] Marsh D (2002) Membrane water-penetration profiles from spin labels. European Biophysics Journal 31:559-562

[76] Mitra K, Ubarretxena-Belandia I, Taguchi T, Warren G, Engelman DM (2004) Modulation of the bilayer thickness of exocytic pathway membranes by membrane proteins rather than cholesterol. Proceedings of the National Academy of Sciences 101(12):4083-4088

[77] Bermúdez H, Hammer DA, Discher DE (2004) Effect of Bilayer Thickness on Membrane Bending Rigidity. Langmuir 20:540-543

[78] Gao L, Shillcock J, Lipowsky R (2007) Improved dissipative particle dynamics simulations of lipid bilayers. The Journal of Chemical Physics 126:015101

[79] Kučerka N, Gallová J, Uhríková D, Balgavý P, Bulacu M, Marrink SJ, Katsaras $J$ (2009) Areas of Monounsaturated Diacylphosphatidylcholines. Biophysical Journal 97:1926-1932

[80] Castillo N, Monticelli L, Barnoud J, Tieleman DP (2013) Free Energy of WALP23 Dimer Association in DMPC, DPPC, and DOPC Bilayers. Chemistry and Physics of Lipids 169:95-105

[81] Kiselev MA, Zemlyanaya EV, Ryabova NY, Hauss T, Almasy L, Funari SS, Zbytovska J, Lombardo D (2013) Influence of ceramide on the internal structure and hydration of the phospholipid bilayer studied by neutron and X-ray scattering. Applied Physics A 116(1):319-325 
[82] kB1 and cO2 backbone flexibility. MP4 clip. https://w-

hs.sciebo.de/s/OXJdy4we5qgufr7. Accessed 23 February 2021.

[83] kB1 membrane interaction. MP4 clip. https://w-

hs.sciebo.de/s/mPI2E2PEtkqtWv6. Accessed 23 February 2021.

[84] Nourse A, Trabi M, Daly NL, Craik DJ (2004) A Comparison of the Selfassociation Behavior of the Plant Cyclotides Kalata B1 and Kalata B2 via Analytical Ultracentrifugation. Journal of Biological Chemistry 279(1):562-570

[85] Rosengren KJ, Daly NL, Harvey PJ, Craik DJ (2013) The Self-Association of the Cyclotide Kalata B2 in Solution is Guided by Hydrophobic Interactions.

Peptide Science 100(5):453-460

[86] Quantitative cyclotide-membrane interaction analysis. MP4 clip. https://whs.sciebo.de/s/VGwXDawV4a05JUC. Accessed 23 February 2021.

[87] kB1 "hydrophobic patch" mutants membrane interaction. MP4 clip. https://whs.sciebo.de/s/JyQNKNdtVqbYKyi. Accessed 23 February 2021. 